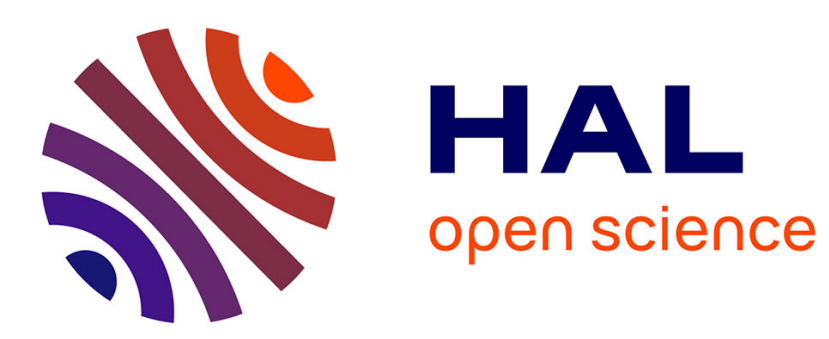

\title{
Standardization of Protocol Design for User Training in EEG-based Brain-Computer Interface
}

\author{
Jelena Mladenović
}

\section{To cite this version:}

Jelena Mladenović. Standardization of Protocol Design for User Training in EEG-based BrainComputer Interface. Journal of Neural Engineering, 2020. hal-03133558

\section{HAL Id: hal-03133558 \\ https://hal.inria.fr/hal-03133558}

Submitted on 6 Feb 2021

HAL is a multi-disciplinary open access archive for the deposit and dissemination of scientific research documents, whether they are published or not. The documents may come from teaching and research institutions in France or abroad, or from public or private research centers.
L'archive ouverte pluridisciplinaire HAL, est destinée au dépôt et à la diffusion de documents scientifiques de niveau recherche, publiés ou non, émanant des établissements d'enseignement et de recherche français ou étrangers, des laboratoires publics ou privés. 


\title{
Standardization of Protocol Design for User Training in EEG-based Brain-Computer Interface
}

\author{
Jelena Mladenović ${ }^{1,2}$ \\ 1 Inria Bordeaux Sud-Ouest, LaBRI (CNRS, Univ. Bordeaux, INP), France \\ 2 DyCog, CNRL, Inserm, Université de Lyon U1028, France \\ E-mail: jelena.mladenovic@inria.fr
}

\begin{abstract}
Brain-computer interfaces (BCIs) are systems that enable a person to interact with a machine using only neural activity. Such interaction can be non-intuitive for the user hence training methods are developed to increase one's understanding, confidence and motivation, which would in parallel increase system performance. To clearly address the current issues in the BCI user training protocol design, here it is divided into introductory period and $\mathrm{BCI}$ interaction period. First, the introductory period (before BCI interaction) must be considered as equally important as the BCI interaction for user training. To support this claim, a review of papers show that BCI performance can depend on the methodologies presented in such introductory period. To standardize its design, the literature from human-computer interaction (HCI) is adjusted to the BCI context. Second, during the user-BCI interaction, the interface can take a large spectrum of forms (2D, 3D, size, color etc.) and modalities (visual, auditory or haptic etc.) without following any design standard or guidelines. Namely, studies that explore perceptual affordance on neural activity show that motor neurons can be triggered from a simple observation of certain objects, and depending on objects' properties (size, location etc.) neural reactions can vary greatly. Surprisingly, the effects of perceptual affordance were not investigated in the BCI context. Both inconsistent introductions to BCI as well as variable interface designs make it difficult to reproduce experiments, predict their outcomes and compare results between them. To address these issues, a protocol design standardization for user training is proposed.
\end{abstract}

Keywords: Brain-computer interfaces (BCI), electroencephalography (EEG), perceptual affordance, human-computer interaction (HCI), user training, protocal design, standadization

Submitted to: J. Neural Eng. 


\section{Introduction}

Technological advancements, notably machine learning [1] and open-source signal processing platforms (openViBE [2]) enabled systems such as BrainComputer Interfaces (BCIs) to increase their usability for a vast population. Thanks to the high temporal resolution of electroencephalography (EEG) and its portability, it is the typical device used to measure neural activity in BCIs. Through neural regulation, a BCI can be used for (1) medical purposes that include control [3], communication [4] or motor rehabilitation [5]; (2) implicit environment adaptation for better user experience in gaming [6] or safer piloting [7]; and (3) cognitive restoration using a therapeutic (neurofeedback) application [8].

BCIs contain closed-loop interactions that include: 1. a person who has short/long term intentions/goals for using the system, 2. a machine that captures, interprets and classifies its user's cerebral activity, and 3 . an interface (displayed on a screen, audible on speakers and/or as a tangible device) that is controlled by the machine, and perceived by the user, enabling the two to interact.

BCI performance (often measured with classification accuracy) is generally not high or robust enough to be safely used outside of controlled environments [9]. Due to the abundant inter (e.g. brain morphology) and intra-subject (e.g. attention drops) variability among others, that deteriorate the decoding of brain signals and system performance, it seems difficult to create a unique BCI system with high performance and usability for everyone [10]. Furthermore, to achieve moderate to high performances, the user is supposed to understand the task at hand and produce distinct and stable neural signals [11]. For that reason, some proposed user training in order to assist the user in learning to interact with a BCI, or acquiring the so-called "BCI skill" [11]. Spontaneous BCI paradigms, e.g., sensorimotor rhythms (SMR), are found to be more difficult to control (higher "BCI inefficiancy" [12]) than the eventrelated potentials (ERP) or steady-state evoked potential (SSEP) paradigms, as they require higher cognitive abilities such as mental object rotation, mental calculation, kinaesthetic imagination or visualisation etc, all followed by attentional focus [13].

In this paper, user training is referred to as methodological approaches tailored to the user to gain enough skills, understanding, confidence, acceptance, motivation, and so on, enabling them to use the system on their own (with high performance), outside the laboratories. Not to be confounded with the machine training and testing, in which the machine acquires enough training (EEG) data from the user as part of machine learning in order to perform testing or classification of newly acquired neural data. Although rare, BCI user training can also be useful in ERP (e.g. P300) paradigms, as attentional focus and motivation can improve with the repetitive interactions with BCI, for both visual $[14,15]$ and auditory [16] modalities. On the other hand, there seems to be no special need for SSEP high attention or effort to accomplish the task, as showed for visual SSEP (SS-V-EP) in [17]. However a recent study revealed that familiar background music might have positive effect on SSVEP performance [18].

A protocol design is a set of choices or rules the experimenter has to make when conducting an experiment to evaluate a hypothesis, or a trainer, instructor or designer could face when training a BCI user. The protocol can contain many logistic challenges such as the duration of the training, location and its physical conditions, among many. It is important to acknowledge that BCIs are prone to interference from other electromagnetic sources [19]. Such logistic challenges are not detailed here, nor ethical approvals, hypothesis formulation, choice of population number and properties, statistical tests to be used (preferably blinded), and other (in the case of experimental protocols). A pedagogical guide for designing a BCI experiment in general and assessing its validity can be found in [20]. A consensus providing a clear checklist was recently proposed to guide correct reporting and experimental design of neurofeedback [21].

This paper brings another aspect of design standardization that includes the user introduction to and interaction with an often stimulation-rich BCI environment that unaccountably modulate neural responses and performance. We reach such standardization through an exhaustive literature review from BCI as well as other fields such as cognitive psychology, and human-computer interaction (HCI).

\subsection{Related work on BCI User Training}

Abundant research was done so to improve BCI performance and usability through user training by addressing user skills, learning, contextual states, i.e., 
states that relate to the context or task at hand, such as confidence to accomplish the task, feeling in control, sense of agency, workload, attention, understanding, motivation, and so on. Here, the existing BCI user training is arranged by the moment it is provided, i.e., before/during or after the user-BCI interaction. With the temporal division of user training proposed for a systematical review of related work, it becomes clear that, when designing the protocol for user training to improve performance, one aims to:

(a) Develop user skills long before the interaction, by mainly training the user multiple sessions before the interaction with a BCI. BCI performance predictors, i.e., human factors that relate to or predict performance are often studied during such training. Skills investigated for SMR paradigms are for instance: mental object rotation, visuospatial memory [22], muscle relaxation [23], mindful meditation [24], sport, playing an instrument or gaming [25], and so on. Moreover, stable predictors such as handedness [26], personality [27] or temperament [28] can provide insight into individualized interface and task designs. Also heart rate variability showed to relate to ERP performance [29], as well as gamma oscillations (attention span) for SMR [30]. Although [31] have questioned the existence of reliable predictors. Indeed, the differences in the experimental protocols, especially in SMR paradigms might prevent reliable comparisons and predictions [32], and reproduction of results.

(b) Prepare user contextual states right before the interaction or during interaction breaks, by providing various types of assistance such as explanations about the BCI functionalities and the task goals. Displaying raw EEG signals to the users possibly increased their sense of agency in [33], while showing brain activation map in realtime was suggested in [34]. Placing personalized physical objects in front of users can increase feeling of control and performance [35]. Physical training together with imagined motor training before the interaction enhanced Event-Related Desynchronisation [36]. They also used explanatory videos demonstrating the performed task in first person. Explaining the importance of user effort for saving/improving lives of severely injured patients increased user's intrinsic motivation through the 'willingness to participate in a task' [37], it showed useful especially for more empathetic BCI users [38]. The BCI users can be instructed to perform mental tasks such as kinaesthetic (a feeling of a movement) versus visual (first/third person) motor imagery [39]. They can be instructed to add emotional values (e.g., to think positively) for neural regulation which improved alpha peaks up-regulation [40, 41] while it hampered modulation of slow cortical potentials [42]. Users choice of imagined movements can be further guided with more specific instructions, e.g., by asking user's preferred or habitual movements [43]. Specific (e.g. "squeeze a ball") instructions be it personalized (user's choice of movement) or not, yielded higher performance than non-specific instructions, e.g. to "imagine a hand movement" [35].

(c) Improve BCI-user interaction, e.g. by gamifying the interface to improve user experience [44]; or immersion with virtual reality (VR) environments [45] (using game-like context [46] and or proprioseptive, "embodied" feedback [47, 48], with multimodal output [49]). Namely, proprioseptive multimodal feedback (touch and visual stimuli) when compared to non-proptioceptive feedback, showed a significant increase in performance [50]. Other tasks included a social (competitive/collaborative) event to increase the experience and motivation [51, 52]. Optimistic feedback displaying only correct results [53], or biased feedback depending on performance [54] was to possibly increase confidence, sense of agency through body-ownership illusion [55]. Also, motivating music was played in the background as tentative to increase flow (a state of optimal immersion, pleasure and confidence) [56] that positively related to performance. Sound congruent with the motor imagery of feet was proposed to be more intuitive to its users, i.e., hearing oneself walking on gravel [57].

Game-like interfaces for ERP [58] or SSEP paradigms [59], were also proposed, summarized in $[60,61]$ to increase user experience; $3 \mathrm{D}$ over 2D environments produced higher performances and elicited higher amplitudes of P300 waveforms [62]. VR environments showed more intuitive, with objects being the target or congruent to the selection task [63, 64], same for Augmented Reality environments [65]; while smiley faces [66] and familiar faces [67] or one's own face [68] showed to be more easily recognized by users than flashing colors, and produce stronger P300 amplitudes. Same applied to auditory P300, when hearing one's own name versus random words [69]. Items flashed far both spatially and temporally [70], intelligent (but not data-dependent) order of stimuli apparition [71]; inter-symbol distance, symbol size, contrasted foreground and background colours [72] produced clearer P300 potentials. Moreover, word auto-completions were developed for faster P300 spelling [73]. For SSVEP, "using distant targets of different colors seems to lead to the best and more robust performance in all end use contexts" [74]. In addition, SSVEP does not require a high visual attention or cognitive workload [17]. Thanks to this, SSVEP can be used simultaneously with another paradigm, such as motor imagery [75], with almost equal performances as compared to using each 
paradigm separately SSVEP and MI [76] or SSVEP and P300 [77].

Adaptive Interaction. The interface (task design) can adapt to its users, as the difficulty that was gradually, within sessions, increased to improve user learning, from $1 \mathrm{D}, 2 \mathrm{D}$ to $3 \mathrm{D}$ control [78], while the speed increased difficulty within trials [79]; or the number of flashes reduced in each sequence [80]; also, the difficulty varied in real-time to increase flow state [56]; or interaction "froze" when high attention level was detected [81]; and attention diversion during motor execution was regulated with auditory oddball paradigm [82]. Users' skills can be assessed (before interaction) in order to design adapted BCI tasks and increase performance [83]. Such adaptation can be automatic to fit the user capacities, showed in a simulation [84]. Finally, paradigms can be switched between or used multiple at the same time, i.e., hybrid BCIs [75, 85].

Studies from (c) address the design of the interface and the interaction processes that account for human psycho-physiological factors and may increase the performance. All these choices made for the user$\mathrm{BCI}$ interaction, are denoted as interaction design. On the other hand, regarding only the interface how to convey the task purpose through it, and what form/modality should it take etc., here is referred as task design. This topic will be developed further hereinafter.

(d) Improve user motivation with a reward system after BCI interaction, e.g., the use of smileys to reward user effort [86], monetary rewards to increase extrinsic motivation [87] etc.

\subsection{Inconsistent BCI User Training}

Non systematically, experimenters used various motivational, methodological and pedagogical tools in different modalities (oral, textual, video, tangible etc.) before/during the interaction to improve user's contextual states (understanding, confidence, motivation, attention, workload, etc.). Some even omitted to describe the instructions given to the users in their protocol designs. Most of these studies have not followed a standard way to represent/ explain the task and BCI underlying functionalities, which means that it can vary greatly between protocols. This further implies that the performance can vary between the protocols due to such difference in instruction presentations, or even with the experimenter's gender [88]. While most sought to design game-like user training during BCI interaction, they have not accounted for the many influences the stimuli (within the interface) can have on neural activity. Especially games which are rich environments can evoke various reactions that are unaccounted for. Namely, various perceptual distractors showed to increase cognitive load which negatively correlated with performance (for low performance subjects) especially for motor imagery BCI [89, 90]. Furthermore, adding smileys expressing different emotions with flickering lights as redundant visual feedback positively influenced motor imagery performance [86]. This shows that comparisons between such different designs can be difficult, especially when some descriptions are omitted. It is worth noting that for SSEP, there were attempts to compare various designs (light, colour etc.) [91]. However, recently the use of background music showed to influence performance in SSVEP only if familiar to users [18], and not otherwise [92]. This indicates that there is also a need for task design standardization, even in SSEP paradigms which typically do not require high cognitive effort or attention [17].

\subsection{User Training Standardization}

A reward is clearly significant for performance and motivation, however its presentation to the users might not be of such importance. Thus, the protocol design for user training, in this paper includes: (1.) the user introduction right before or assistance during interaction (described in (b) above), and (2.) the user-BCI interaction itself, since the lack of a clear methodology in both can cause differences in performance (described in (c) above). I present a literature review of existing BCI training methods to support the need for standardization. Doing so, some recommendations may occur for "optimal" methodologies in user training, although it is not the goal of this paper.

The goal of this paper is to make clear distinctions of methodologies that were or can be used for (1.) user introduction to and (2.) interaction with a BCI, the second being presented through (3.) a task design. That is, a standardization is proposed for a protocol design for BCI user training.

(1.) To highlight the importance of user introduction to BCIs, and guide its standardization, a review from Human-Computer Interaction (HCI) user training is presented, as they confronted similar issues with computer acceptance and learning many years before BCI community did.

(2.) For the interaction design standardization, it is impossible to create precise scenarios of protocols that would fit every user psycho-physiological profile, as there are countless possibilities, and not an exact, optimal solution for now. However, here a standardization of the designer's/experimenter's choice flow is proposed, i.e., systematic choices or thought processes to be followed during an interaction design. It is a tool that reveals the BCI interaction factors to be accounted for, and in what order. As building blocks, existing conceptual BCI interaction models are used 
which contain the machine pipeline [93], user model [94], and task model [95].

(3.) The task designs are addressed for their implication on forming specific neurophysiological reactions. A new task category is proposed in order to enable its standardization, such as the task form (e.g. $2 \mathrm{D}, 3 \mathrm{D}$, continuous feedback), as its effect on neural activity is least studied in BCI. The proposed subcategories of the form are drawn from the literature in perceptual affordance [96], i.e. perceptual information of an object implicitly suggesting a set of possible actions to be performed on that object. With different properties (size, shape, location etc.), the object can afford different neural reactions (cf. section 4). Hence, the task design form is standardized by the effects it can have (afford) on neural activity.

When creating training protocols, I invite experimenters, and designers to describe which category their instruction, interaction/task designs belong to. That way, by knowing such category, possibly one would better anticipate the experiment outcomes. Additionally, others would be able to reproduce or compare the results more easily.

\subsection{Paper Structure}

The BCI instructions before/during the interaction, or called here, the introductory user training in (Section 2), followed by an overview on Human-computer interaction (HCI) training in (Subsection 2.1) inspire the proposal for the introductory user training standardization in (Subsection 2.3). The interaction design (Section 3) contains explanations about the BCI task and task models (Subsection 3.1), followed by the standardization of the experimenter/designer's choice flow in (Subsection 3.2). Thanks to the literature in perceptual affordance in (Section 4), a standardization of BCI task design is proposed in (Subsection 4.4).

\section{Introductory BCI User Training}

As computers are used for a few decades now, there are many methods developed as part of HCI user training, to teach users to develop the right techniques when using a computer. The BCI community can get inspirations from the HCI task models and user training for designing more rigorous BCI user training.

\subsection{User Training: Lessons from HCI}

Human learning is often performed through the creation of mental models of the system one interacts with. Mental models believed to originate from the book The Nature of Explanation [97], a concept used by cognitive psychologists [98] and HCI researchers [99] among others, are internal representations of the world that humans create in order to make meaning, anticipate events and act to minimize anticipation error [100]. To assure a correct formation of one's mental model of a computer system, HCI community proposes various task models for user training. Already by the 90's, a large number of user training for computer systems were proposed. From an overview of the literature, there are three main types of user training, which use: conceptual models [101], procedural models [102] and interacting with the system [103]. These models are not to be confounded with user mental models. Note that these models can assist in forming mental models which enable users to perform mental actions before actually performing them. Such kind of prediction and reasoning can increase learning, thus represent an essential part of training.

Conceptual models represent manuals (tutorials) that provide an understanding of the underlying processes of the system. It can contain (i) word analogies to describe a new concept by comparing with another familiar one; or (ii) abstract descriptions, such as charts, diagrams, e.g., an inverted tree with a root as a directory and branches for files hierarchy, etc. [104]. Using analogies for conceptual models can form wrong mental models in novices, as different persons might project their own experience to the metaphor, and have a wrong action in mind when confronted with the task [105]. On the other hand, abstract metaphors can be difficult to comprehend to some users, for instance for low-visual and low-abstract persons [106].

Procedural models represent a "how-to" tutorial, describing step by step procedures to follow, in order to achieve a goal. They do not contain any information about the system structure or its components. The most known procedural models are GOMS (Goals, Operators, Methods, Selection rules) [102]. To accomplish a goal, one should choose a set of operators leading to various system states. For instance, to copy paste a text, one needs to perform a left-mouse click to select the text, then press Ctrl-C on the keyboard to copy,.. press Ctrl-V to paste, and so on. It can be useful when the system is simple to operate. Specific task instructions have shown to be better than general instructions, as such they create less confusion. However, when it comes to transferring knowledge to a slightly different task or confronting with errors, these models are sub-optimal [107].

Training through Interaction is to learn through trial-and-error while interacting with the system directly. Such training models often form incomplete mental models in novices leading to error and frustration [103]. However it is an important part for enhancing existing mental models that were for instance already created using conceptual models [107]. In [107] authors explain the importance of training 
models for correct mental model formation, which can assist users to reason and understand better the behavior of the system. They show that mental models formed from conceptual models require deduction to form specific procedures, while procedural require a high level of abstraction to form mental models. The latter is typically much more difficult to achieve. Also, they explain how most manuals that can be found are procedural ones, and seem to be preferred by users as they produce rapid solutions to attain short-term goals.

\subsection{User training in $B C I$}

The ability of users to understand computer systems affects their acceptance and utilization [107]. It was shown that fear of technology negatively influences BCI performance [108]. It is possibly because users lack understanding of the underlying processes of a BCI. In that sense, BCI users could benefit from conceptual models when engaged in training. A majority of studies focuses on the Interactive BCI user training, i.e., the user is supposed to learn through trial-and-error, using non-explanatory cues and feedback, particularly important in SMR paradigms [11]. However, differently from a computer system where users perceive a direct effect of their conscious, physical actions, BCI users have little control over their actions and rarely create a direct link between their mental action and perceptual observation. This often creates a mismatch in what users expect to observe and what they actually observe, leading to a lack of sense of agency or control [33]. Meaning that, standard user training through interaction might not be the optimal solution for BCI users. As mentioned above, even in HCI, such training is shown suboptimal for novice users [103]. Again, BCI training might benefit from the use of conceptual and procedural models to first create a mental representation of a BCI system and then enhance such representation with Interaction. Only a few studies in neurofeedback (NF) have investigated the impact of user mental models on the neural modulation [109]. Namely, the instructions given to the users during the introductory period can impact the formation of mental models, typically addressed in $\mathrm{BCI} / \mathrm{NF}$ as mental strategies. Authors in [109] validated nine categories of SMR mental strategies that include (1) visual or (2) auditory imagination, (3) focus on breathing, (4) on body parts, (5) motivational inner voice, (6) physical relaxation, (7) concentration on task, (8) unrelated thoughts requiring cognition, (9) no strategy (automation). They further show that a user will use two-three mental strategies on average to accomplish the task. Once the user finds the right strategy, he will use it until it becomes natural or effortless, reaching automation, or the (9th) no strategy category.

\subsection{Proposal for Introductory BCI User Training}

Following advice from HCI user training, and gathering the impact different instructions can have on neural modulation (formation of mental strategies), a standardization for user introduction to the BCI interaction is proposed. Figure 1 is further explained as follows.

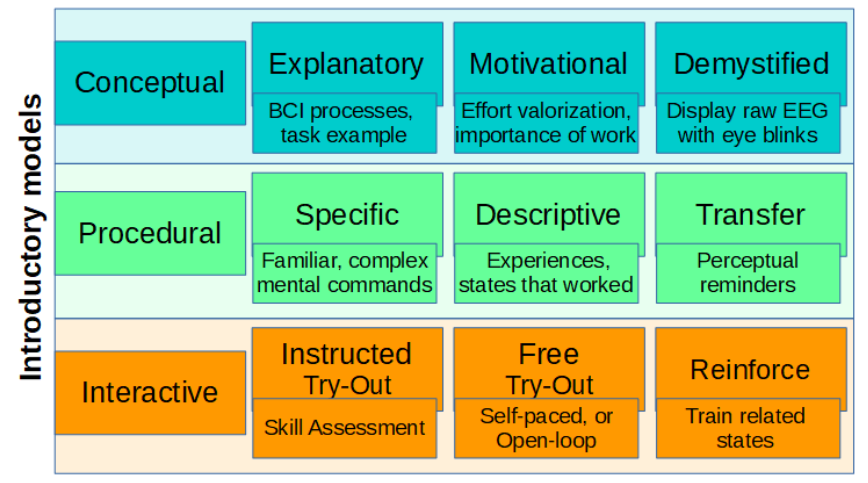

Figure 1. Introductory user training standardization inspired by the training models from HCI literature that would enable a correct formation of user mental models (or known in BCI as mental strategies).

Conceptual BCI user training models could contain a short explanatory video/text/oral presentation briefly describing the concepts and underlying mechanisms of BCI through popular and pedagogic word metaphors, e.g. "your brain is like a muscle that can be trained" [110]. Importantly, to reduce fear from technology brought by media [111], it must be specified that a BCI is not a "mind reader", i.e., it cannot infer one's intelligence, emotional stability, general health, or that it cannot insert thoughts into one's mind, and so on. It could also contain a video demonstrating another person performing the task, as in [36], or a simulation of the task [112]. Let us call this type of conceptual model Explanatory.

The users could be told about the importance of their effort to improve BCI systems which can help countless of people in need, as demonstrated in [37]. This one is denoted here as the Motivational conceptual model.

Another approach could use abstract representations of one's EEG signals in real-time and seeing the effect of eye blinks or teeth clenching, in a time frequency domain [33], or brain map neural activity [34]. Let us call this type of conceptual model Demystified.

Procedural BCI models lack precise howto manuals for forming mental strategies, as for Motor/Mental Imagery. However, we can advise, describe and or even train users outside of a BCI 
context, to be in certain states (cognitive [113], emotional [40, 41] and physical [109]) that have shown favourable for BCI performance, e.g., high attention $[113,114]$, trained with mindful meditation [24]. It is important to note that the "best" user states depend on the neural modulation type or BCI purpose, e.g., alpha up-regulation benefits from an additional emotional (positive) perspective $[40,41]$ while its down-regulation would rather benefit from a cognitive one (attentional focus) [113]. Let us denote this procedural model as Descriptive.

Instructions should be informative, precise and propose specific mental exercises to be imagined, e.g., "squeeze a ball" [11, 35] potentially ones that include a complex movement i.e., an imagined movement that includes multiple body parts [115]. Importantly, specific strategies that are performed in everyday life or learned before, i.e., familiar activities, should be proposed [115, 116]. On a side note (not tested in BCI yet), statements which include "do not think of" an exact action should be avoided, as it will attract the mind into thinking precisely that action, known as the ironic process theory [117]. Instead, the undesired mental strategies should be left rather abstract. Let us denote it as a Specific procedural model.

Physical together with mental training on objects of choice [36] should be provided, or only observation (if physical action is not possible) of the personal object [35]. They can be present during the interaction, as perceptual reminders that could assist in the creation and consistency of the mental strategy. This way one can Transfer the trained mental strategy onto the BCI application.

Interactive BCI models were either used for user skill assessment in order to personalize (adapt) the future task, and increase performance [83], or for training a useful skill or state, eg. tactile selective attention before the motor imagery task [118]. [80] used an adaptive P300 BCI game to increase visual attention, it could be also considered in the context of an introductory BCI user training. Furthermore, interactive BCI models can be self-paced BCI try-out without any cues, but with feedback [119], or with cues but without any feedback, called open-loop [120].

Each designer/experimenter should choose and describe one or a combination of models depending on the purpose of BCI application and paradigm. The procedural model is more likely to be used for Spontaneous (like SMR) paradigms, while conceptual and interactive could be useful for all paradigms.

The protocol design in this paper includes the introductory period, as well as the interaction with the BCI. In the following, the interaction design is described starting with the conceptual models for BCI interaction.

\section{Interaction Design}

To comprehend the BCI interaction systematically, it is simplified through the use of conceptual models. The most used conceptual model is the system pipeline which explains mainly the processes the machine performs [93]. Recently a simplified representation of the user's psycho-physiological factors that relate to BCI performance became a relevant part of the BCI interaction model [94]. Finally, when addressing adaptive BCIs, a task model was added as a link between the user and machine adaptive processes, all arranged by the degree of changeability in time [95]. In this paper, the interaction model from [95] is extended and used to standardize the interaction/task design, see figure 2 .

When designing the user-BCI interaction, the questions to be answered are the following (a step-bystep description of figure 2):

1. Human (user model) - Which human factors (predictors) to account for, from stable characteristics such as age, through personalities, skills, or contextual states, to short-term reactions to stimuli? As in [95] the factors are arranged from stable to very changeable, from top to bottom. The information on human psycho-physiological factors can be used to: 1 . initially design the task by (without changing it in time), e.g., according to some stable factors which do not change during a BCI session; 2. adapt the task and system pipeline to, e.g., according to contextual states; or 3 . simply pay attention as confounding factors. These information, an experimenter usually acquires from various questionnaires before and or during the interaction. Although, the community strives to find robust neural markers of states (e.g. attention or workload) as more objective measures than questionnaires [121].

2. Machine (pipeline model) - What hardware for signal acquisition to choose (e.g., active/passive, dry or with gel electrodes); and what software to use (e.g., OpenViBE for real-time signal processing, feature extraction and classification), for such information, see [122]. How can a machine account for user psychological/cognitive factors? For instance, in [120] they chose a fixed classifier to enable a steady user learning. On the other hand, some proposed co-adaptive machine learning for the same purpose [123]. Which pipeline element should adapt during the interaction, and how often, see suggestions in [95, 124].

3. Interface (task model) - What paradigm, strategy, exercise to train the user in, and for what purpose? How to present it, in which form and modality? What type of assistance during the 


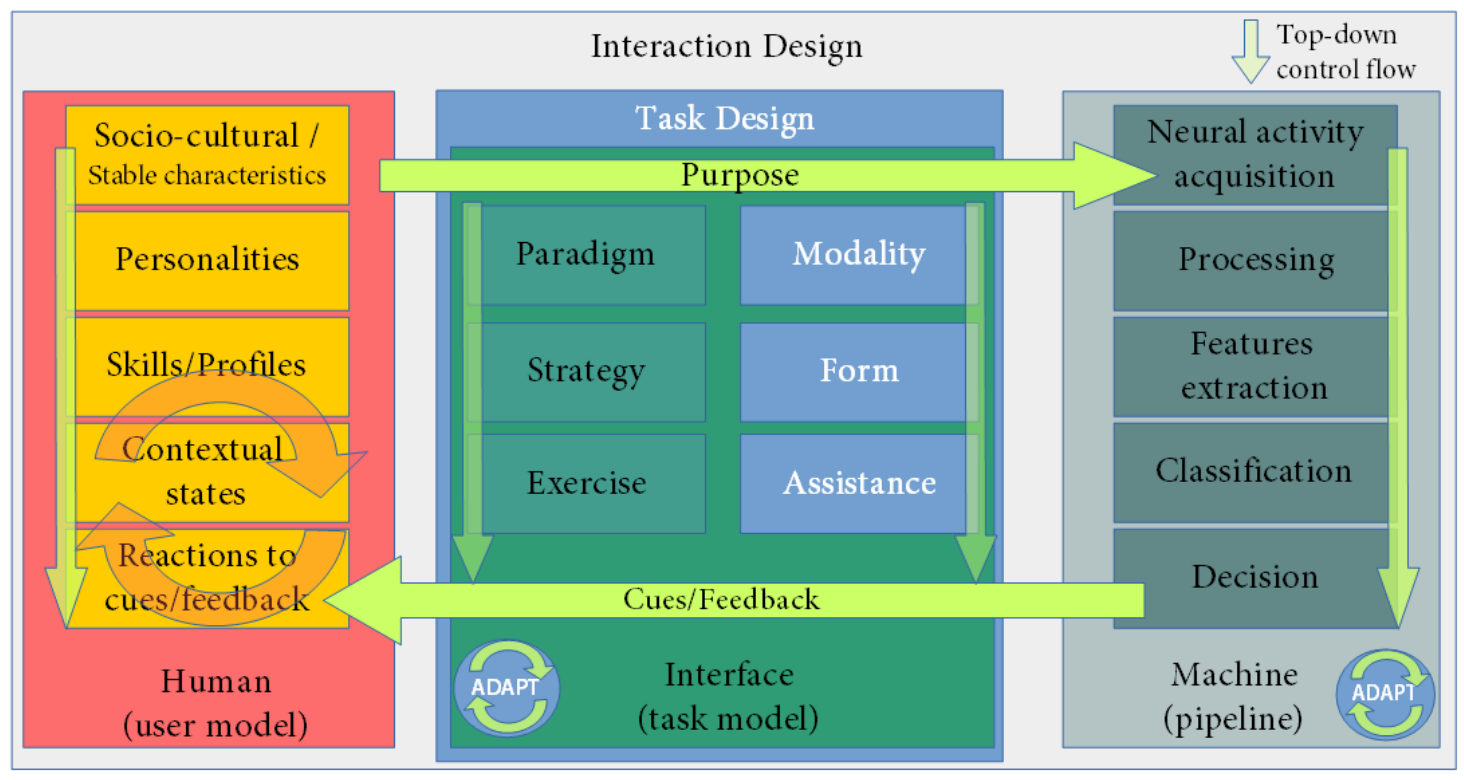

Figure 2. Interaction Design (extended from [95]) that contains: task and user models (arranged top-bottom from long to short term features) and the well-known BCI pipeline model. The task design here is the manifestation of the task model through modality, form and assistance.

interaction to provide, e.g. should the feedback/cues be realistic or biased? Additionally, should it be adaptive in real time or within some periods of time? As the task model is by itself a recent approach, more details are provided in a dedicated subsection bellow.

Adaptive interaction. Adaptation can appear within all 3 models of interaction. First of all, users naturally adapt (brain plasticity [15]) but not necessarily learn [124]. Also psychologically, contextual states can vary during a BCI run, as a change in attention, workload, mood etc [95, 125]. If one wants to adapt the pipeline or interface to human factors, they should consider the degree of changeability or stability in time [95]. Namely, the machine nor interface should not change faster than one human feature would. For instance, if the machine adapts to increase user learning, and it adapts too fast before anything was learnt by the user, then it might prevent the user of making any effort and impede his learning [126], as shown mathematically in [127]

\subsection{Task Model and Design}

BCI Task. The term task is here defined as what is required from a user to accomplish a successful interaction and reach her purpose (by achieving many short term goals). The task is conveyed through an interface taking for instance many modalities, e.g., visual, auditory, haptic. For instance, as part of user introductory training, the task would be introduced as such: "look at the computer screen, when you hear a beep and see a flag on the left side of the ski course, imagine to move your left hand in order to control a virtual penguin to the left" or "letters on the screen flash repetitively, focus your visual attention on the letter that you wish to spell, count the times it flashed; once it is done it will spell a letter". Let us analyze these sentences.

1. hear a beep, see a flag... - auditory and visual cues within the interface (screen);

2. imagine to move - it is a motor imagery belonging to the SMR paradigm. The strategy can involve a visual imagination in third or first person, a kinaesthetic sensation, and so on;

3. left hand - includes a specific hand movement exercise e.g., grasping, waving, typing etc.

4. to control a virtual penguin - the expected visual feedback to the motor imagery (short term goal).

Note that this is an example of a synchronous SMR, for which the motor imagery exercise is being guided and paced by visual cues. However, the SMR can be self-paced, without any cues [128].

Or:

1. letters flash ... - visual cues (stimuli) within the interface (the screen);

2. focus visual attention - ERP paradigm, with the P300 visual attention strategy;

3. count the times it flashed - precise exercise;

4. letter you wish to spell - expected visual feedback to the P300 responses (short term goal).

This clearly shows that the term task supports many notions. It contains goals that drive actions which are: triggered by cues (stimuli), belong 
to a specific paradigm, strategy, exercise, and evaluated/reinforced by feedback. The task model from [95] is defined as the ensemble of tasks a user goes through when interacting with a BCI, including:

1. Purpose for using a BCI (e.g. communication, neuro-rehabilitation), that contains short-term goals for accomplishing the task at hand.

2. Paradigm used (e.g. ERPs or SMR), or a combination of paradigms, i.e., a hybrid BCI which can also include other physiological/behavioral input such as eye tracking or mouse clicks etc [85].

3. Strategy that is twofold: one represents the general neural modulation that depends on the paradigm, e.g. P300 response or Motor/Mental Imagery; while the other represents individual interpretation, mental execution of the task, e.g. motor imagery can be performed kinaestheticaly or visually [129] with complementary sound imagination or motivational inner voice, and so on [109].

4. Exercise, depending on the strategy, it can be e.g. imagination of a precise limb moving, object rotating, a precise calculation, or counting flashes etc. For instance, "squeeze a ball" motor imagery yielded higher performance than a non-specific one, to "imagine a hand movement" [35].

5. Cues indicate which exercise is to be performed at the following instant, or serve as stimuli which elicit neural potentials (SSEPs or ERPs); It is worth mentioning that in gamified interfaces there can be many stimuli present that are irrelevant to the task but induce immersion, e.g. background music [56]. The effects of such stimuli are yet to be clarified.

Feedback, is a mapping of neural activity (extracted EEG features) onto perceptual events in minimal delay depending on the sampling rate of signal acquisition, time windows used for signal processing, and so on. There are examples in which it does not map neural activity in almost real-time, but in some way evaluates/reinforces neural self-regulation, often after a trial or run is over [86, 130]. zich2015real

Interface. The BCI task is conveyed through the interface that can be considered as a medium of interaction taking many forms, modalities and types of assistance. To design an interface is referred to as the Task design, see figure 2. Here, the extension of task model from [95] contains modality, form and assistance, as well as the cues and feedback. Namely, the interface can vary in:

1. Modality, that is visual, tactile or haptic, auditory, other, or their combination. Multimodal information has shown useful for attention allocation and reduction of cognitive load, as it is similar to real-life situations [47, 131]. Interestingly, a robotic hand that touched the user's hand showed higher sensorimotor activation and feeling of control, versus hand on screen $[50,132]$. Cues in different modalities such as visual arrows pointing left and right, or sound of words "left" and "right", and the two combined, did not change classification accuracy but did modulate neural patterns [133].

2. Form, depending on the modality, it can be in $1 \mathrm{D}, 2 \mathrm{D}, 3 \mathrm{D}$, with continuously or discretely moving/appearing objects in various locations and timing, different colours, shapes and sizes, or melodic sounds or beeps, etc. This element will be enriched with precise sub-categories, once the notion of perceptual affordance [96] is introduced hereinafter.

3. Assistance, depending on the modality and form, it can appear either within the interface through task difficulty [78], distractors [89, 90], or positive feedback [53], word auto-completions [73]; or externally through social presence (multiple users, an encouraging experimenter $[51,52]$ or artificial agent [134], or through methodological tools for individualized training (see Subsection 1.1 on related work). Note that the assistance can overlap with the methodologies from the introductory period (before the interaction with BCI) as they can occur during the interaction (task) breaks as well.

Furthermore, the task design can be adaptive in modality (e.g. switch to reduce cognitive overload [11]), adaptive form (e.g. change from $2 \mathrm{D}$ to $3 \mathrm{D}$ [78]), and adaptive assistance, e.g. adaptive feedback bias [56] or data-driven cues \& feedback [126, 135].

\subsection{Interaction Design Choice Flow}

The interaction and task design is the set of choices an experimenter/designer makes for the task, user and pipeline models. The flow of choices is essentially driven by the purpose of BCI application for the user as well as user psycho-physiological factors.

For instance, for the purpose of communication, an experimenter would generally choose an ERP paradigm, with a P300 strategy that follows the well-known "oddball" paradigm, presenting the flashes spatially and temporally as further as possible [70], using famous/familiar faces [67] and smileys [66], 3D instead of 2D grid of letters [62], objects congruent to selection task [60], etc; and X-dawn spatial filter [136] as part of pipeline factors. All are chosen according to the user psycho-physiological factors, e.g., to detect and increase the P300 potential, as well as user experience and motivation. The effects different stimuli can have on EEG signals of an ERP task have been explored in order to produce higher performances or bit rates. They serve as an example, providing insight or guidelines for optimal ERP task designs. Hence, the choice of the task design would depend on the BCI purpose, and on established 
neurophysiological reactions to external stimuli. On the other hand, for the purpose of control (such as controlling a wheelchair [137]), one typically chooses the spontaneous paradigm, using a Motor or Mental Imagery (MI) strategy. However, the task design is less methodological than for the ERPs, especially because the influence of the stimuli on neural activity is less evident. Nevertheless, one would focus on user psychological states such as motivation, confidence and so on, when designing the BCI interface. The typical solution is the use of game-like contexts, however they are rich in stimuli, or perceptually complex, and as such can provoke various neural modulations that are unaccounted for or undesired.

In the following, the issues of the BCI task design is addressed through the review of perceptual affordance. Although there is a tangible affordance $[138,139]$ mostly visual and auditory modalities are detailed as they are most common in BCI.

\section{Task design: Lessons from Perceptual Affordance}

Perceptual affordance [96] is a theory that assumes that the mere perception of an object leads to the "mental activation" of possible actions one could perform on this object. For instance, an object such as an eyebrow pinch provides perceptual information of the possible actions that can be performed on it, as picking it up and pinching it (of course given the observer's experience and socio-cultural background). Such perceptual information actuates in the observer a finite set of possible actions that can be performed. In this theory, perception and action are intrinsically linked, in a continuous feedback loop. As Gibson argued, "We must perceive in order to move, but we must also move in order to perceive". Every action provides feedback about the just-performed movement and generates information that can be used for guiding the next movement [140].

\subsection{Visual affordance}

Behavioral experiments have shown that depending on the size, orientation and location of the object, one can have different degrees of response time, and precision when performing an action [141]. For instance, if the pinch is too small for the size of user's hand, or if it is closer to the non-dominant hand or oriented opposite from the grasp, the response time is lower than if the spatial properties were better suited for that user. Different object shapes that vary in their center of mass can influence a persons gaze and as well as grasp [142]. Interestingly closed contours are more easily memorized and recognized than open ones [143], while curved, spherical versus rectangular, and natural (fabric) versus plastic materials are found to be more aesthetically pleasing [138]. For instance, a plastic box was described as hard and boring, revealing how users describe their perceptual experience of materials and shapes through emotions. Furthermore, when there is fluency and ease in performing an action, users usually attribute a pleasurable feeling opposite from a non-fluent action which brings minor frustration [144]. Certain colours can alter the perception of object's weight, size, temperature, and through word metaphors colour can afford different emotional responses, e.g., blue is cold which has an unfamiliar or sad connotation [145].

Neuroimaging experimental studies show when passively observing manipulable objects versus nonmanipulable ones, there is stronger activation in motor cortex as shown in fMRI [146], also stronger mu desynchronisation over centro-parietal region [147]; and especially within the range of the dominant hand versus non-dominant one [148, 149]. Furthermore, higher motor evoked potentials were found during the observation of graspable objects falling within peripersonal space (i.e., reachable within a hand grasp) compared to the observation of either non-graspable or graspable objects falling within the extrapersonal space (i.e., out of reach or grasp) [150]. More importantly, when the task is to judge the distance of the object, mu desynchronization is strongest when the objects are within the peripersonal space, and diminishes with distance boundaries of peripersonal space, and extrapersonal space [151]. In the same experiment, when the observers were to focus on object identification, while the object location was changed, interestingly, the mu desynchronization was not modulated. This means that the object location can influence motor activation if the observer is not focused on another task. This suggests that "the involvement of the motor network in the processing of visual objects in peripersonal space is not automatic but rather depends on the goal of the perceptual task" [151].

On the other hand, observing actions performed on objects produces the well-known mirror neuron effect that activates motor neurons. Additionally, mu suppression is shown to be significantly stronger when the observation is task-related versus when it is not [152]. In this experiment, subjects were presented a video of repeated mug grasping and in one case they were to count the number of times the mug was grasped (observation is task-related) or the number of colour changes (not task related).

Although when mentioning perceptual affordance one relates to a visual and or tangible experience, however they are explored as auditory/acoustic events as well. 


\subsection{Auditory affordance}

Studies have shown that when hearing sound, participants can, to some extent, determine the properties of the sound source, such as: geometric features of struck bars [153], length of dropped wooden rods [154], the shape of a plate struck by a pendulum [155], the size and speed of rolling wooden balls [156], the size and shape of colliding polystyrene objects [157], shapes made by hand-drawing movements [158], and so on. Furthermore, a listener can determine whether a car is approaching or leaving, in front or behind, far away or nearby, big or small, or whether it is driving on a dry or wet street [159]. Gaver [159] formalized a framework of the "everyday sound" by combining its physical properties and perceptual experiences, and divided them as 1 . vibrating solids (impact, scraping, rolling), 2. aerodynamic (explosions) and 3. liquid sounds (dripping, splashing). He demonstrated that people rarely or almost never confuse these 3 sound sources between them.

Sound can afford action "if the acoustic structure produced from an environmental event is seen as being useful by an agent for the coordination of perception and action..." (within the context of the task at hand); "It is through needing to navigate the urban environment that the sounds of cars emerge as an affordance for navigation" [160]. For instance, participants were able to intercept rolling balls from auditory cues almost perfectly [161], or when hearing the size of a dropped object participants would adjust their grip to anticipate/optimize their grasp [162], or when hearing a certain object material, subjects were inclined to reach for the part of the object made of such material - congruent with the sound [163]. These results clearly show that sound can influence (afford) one's action related (congruent) to the task.

Neuroimaging studies have shown that mirror neurons are not only activated by visual perception of an action but also upon hearing a familiar action as well, for instance hand-clapping [164]. It means that when recognizing the sound, the brain may simulate the action [165]. Sounds associated with bimanual (typing or tearing paper) actions produce greater motor excitability than sounds associated with walking or their control sound (thunder) [165]. In [166] when pianists listened to a familiar piano piece (only which they knew how to play), it activated audio-motor patterns previously found in humans in a variety of action-observation tasks, e.g., watching chord progression on the guitar [167]. High density EEG and mismatch negativity showed that upon hearing a sound related to finger actions evokes activity in motor areas associated with the dominant hand [168].

It is worth noting that music as well can afford action upon a listener. Music affords a "cognitive load off" from the listener as it tells her how and when to move, causing the task at hand (often physical activity) to feel less difficult [169]. This phenomenon happens when the listener is in sync with the music, in which case music rhythm entrains movement [170]. In addition, it is well established that music and sound can have a strong emotional impact on listeners as well [171].

In sum, objects that are congruent with the task, familiar to the users, vary in shape, material, size, orientation and location can afford different action, but also different cognitive and emotional responses.

Nonetheless, in the BCI task one can find an extensive variety of object forms which effects are mostly unaccounted for.

\subsection{BCI task design}

The success of using VR environments for motor imagery BCI $[45,49,55]$ can also be due to the fact that the objects presented are within the peripersonal space, manipulable and congruent with the MI. Also, in MI the objects were easily graspable, i.e., their orientation and size were configured to suit the virtual hands. However, these mentioned effects were not yet evaluated. The congruence to the motor imagery task (hands grasping an object) was compared to the Graz training protocol (a horizontal bar that changes in size) [172] and yielded no difference in performance in [173]. However the authors used the Graz protocol for the calibration phase in both conditions, thus changing the display for the congruent condition impeded the performance, and biased the results. Congruence was also investigated in [174] as a display-control mapping, i.e., left-right versus up-down visual display for the leftright motor imagery control. The congruent (left-right) visual display proved significantly better than the incongruent one; while adding incongruent information can increase cognitive load and errors. Interestingly, game-like and immersive environments could contain such incongruent or irrelevant information to the task, but their positive influence on user motivation might compensate for the cognitive load. Furthermore, gamelike and immersive environments are more aesthetically pleasing which could have also increased implicit acceptability of the system [175]. On the other hand, when comparing vertical bars that change in size with a game (worm race), it did not yield any change in user SMR mental strategies [109]. However the game did not differ in task form, i.e., in congruence, colour, manipulability, familiarity, dimension (remaining in 2D), and so on; and in that way it did not afford different mental strategies or neural patterns. This could imply that by simply gamifying the interface without changing one of the mentioned features of 
perceptual affordance would not have a considerable effect on SMR modulation. In that sense, [176] showed that a car racing game produced significantly higher performance than the extending horizontal bar. I advocate here that it happened not simply because it was a game per se, but because the car was in a 3D environment and it contained a more complex engine (avoiding obstacles to reach end line) than 2D graphics with a simple goal.

Most auditory cues/feedback are simple tones, beeps, or prerecorded short sound samples of instruments e.g., harp and bong [177]. To avoid the overfamiliarization (irritating) effect after many sound repetitions, music generated in real time and tempo modulations were proposed [178]. However, no prior work verified whether users can play the piece or not which showed to determine the activation of mirror neurons (see section affordance). Furthermore, the use of congruent sound versus non-congruent and out-of-pace one, in combination with the visual modality (a robot moving) demonstrated to increase performances in MI of feet [179]. Also, congruent sound of walking on gravel versus non-congruent (complex harmonics, abstract sound) has shown increase in performance [180]. If music is introduced in the background of the task, the experimenter must be aware of the affordance of music on movement or motor planning [169]. As it was suggested in [56] where (unfamiliar) background music disturbed the participants in performing motor imagery of hands as the tempo or rhythm of the piece were not in sync with their imagined movements. Additionally, in an SSVEP game, unfamiliar background music neither disturbed nor was relevant for the participants [92], while the familiar one positively related to performance [18].

The objects represented in a BCI task can differ in size, location (near-far space), orientation, familiarity, manipulability, rhythm, congruence, etc. without any particular verification or control of potential effects on motor/attention activation or modulation. This vast literature indicates that we should design BCI tasks with caution, and that we cannot easily compare results between such different designs. The experimenter should take note on the choices made when creating the task design.

\subsection{Proposal for BCI Task Design Forms}

To assist every BCI experimenter in designing BCI tasks, the task form is divided by the properties and function an object can have, according to the neuroimaging and behavioral evidence of perceptual affordance, and literature from BCI (see figure 3). Note that here, a BCI object can be a cue/feedback or whatever part of interface the users interacts with. The task form can appear in different modalities, while the assistance type could be manifested through various forms as well as modalities (hence the arrow pointing downwards in figure 3 ).

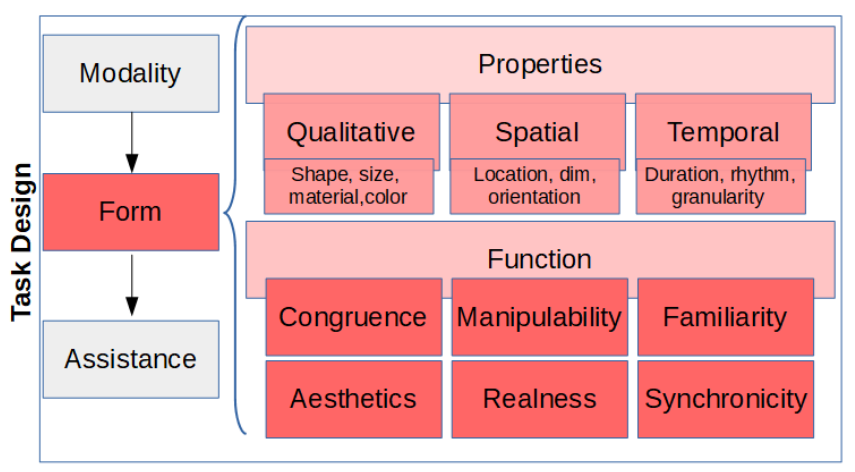

Figure 3. Inspired from affordance and BCI literature, a preliminary proposal for BCI task design standardization, especially focused on the task form (a sub-category introduced in this paper).

4.4.1. Object Properties The perception (affordance) of object properties (in visual, auditory or haptic modality) does not necessarily depend on any previous experience (a priori knowledge), can vary in:

Qualitative properties: Shape, spherical or rectangular/sharp, symmetrical or not, convex or concave, etc; Size, big/small, or fitting in one's hand, etc; Material, it can be rugged or smooth, organic or plastic, etc; Color, the difference in colours of targets in SSVEP [74] influenced performance. The largest SSVEP response was produced by red light at $11 \mathrm{~Hz}$ [91]. In addition, colour can also alter the perception of objects' weight, size and temperature [145]. Contrast is an important feature in SSVEP on computer screens [91], also, contrasted foreground and background influenced performance in P300 [72].

Except for colour (and contrast), qualitative properties were not investigated in the BCI context. Nevertheless, they are important properties according to perceptual affordance.

Spatial properties: Location, objects can be graspable, within the peripersonal space or not (in the VR or haptic modality), they can be situated within the central or peripheral vision, such as center or corners of the screen, they can stand near/far the non/dominant hand of the user etc; one can also distinguish the object location from sound; Orientation, towards the dominant hand to be graspable or if it is a nongraspable/non-manipulable object like a penguin, it can move towards the dominant side or opposite from it, toward/onward the user etc; the sound could move from one speaker to another, oriented towards one ear, 
etc; Dimension, the environment can be in $3 \mathrm{D}$ while the mental exercise in $1 \mathrm{D}$ or $2 \mathrm{D}$, e.g., penguin moves in a 3D space, but the user only performs motor imagery in 1D (left-right, x-axis) [56]. However, dimension can be congruent between the mental strategy and visual display, where for example $1 \mathrm{D}$ environment requires one mental command, and 3D requires at least three which is more difficult to control [78]. Also, 3D environments and VR yield higher performance than 2D, in SMR [45, 176] P300 [62] and SSEP [63, 64]

Except for dimension, object location and orientation were not investigated in BCI context, however they might be accountable for the success of several BCI task designs, notably proprioceptive and VR ones.

Temporal properties: Granularity can be presented as continuous or discrete. High granularity objects appear frequently within a trial (often $\leq 16 \mathrm{~Hz}$ ) being continuous and often smoothed to be perceptually fluent (interpolating temporal gaps between epochs). Typical example is the SMR feedback of a bar that changes in size both continuously and smoothly ("Graz protocol" [172]). Medium granularity objects can appear within a trial $(\leq 1 \mathrm{~Hz})$ but are perceptually discrete, e.g., flickering/flashing for SSVEP/ERP paradigms. Low granularity objects appear once after a trial/run or session is over. The term BCI task granularity is mentioned in [11] where instructional design is recommended for spontaneous BCI user training. Continuous SMR feedback yielded higher performance rates than discrete one [181]. High speed P300 flashing can be tiring, so frequency of apparition was diminished in time [80]. The largest SSVEP amplitudes were observed near $10 \mathrm{~Hz}$, however low frequencies $(5-25 \mathrm{~Hz})$ are subjectively evaluated as more tiring and annoying than higher frequencies $(30-60 \mathrm{~Hz})$ [91]. Granularity can be combined across modalities within trial, e.g. visual as continuous, and auditory as discrete which decreased performance [182]; or visual and haptic in same granularity increased performance [50]. Combining feedback frequency in low granularity can be beneficial for alpha regulation, e.g. after each trial and after a session $[86,130]$.

Duration of cues for MI was standardized to 500 $m s$ in accordance with sensory recognition and reaction time [183, 184]. In P300, the stimuli generally last between $60 \mathrm{~ms}$ (flashing a single item at a time) and around $100 \mathrm{~ms}$ or $200 \mathrm{~ms}$ (flashing a group of items) [185].

Rhythm, SSVEP relies on rhythmical flickering. Depending on the light source, if it is LED, CRT or LCD which have different refreshing frequencies, it is shown that LED produce highest response [186]. The same authors mention that the SSVEP amplitude drops significantly when the refreshing rate increases from 60 to $75 \mathrm{~Hz}$. On the other hand, cues for spontaneous BCI are randomized (not rhythmical), in order to avoid anticipation (movement preparation). In [187], authors found that the involuntary expectations for the approaching cues provoked false control commands during virtual navigation. However, motor anticipation can be beneficial for learning, enabling faster or immediate automatization [188]. Automatization being (in theory) a way neural self-regulation is successfully learned with neurofeedback [109].

Indeed, auditory rhythmic cueing activates motor areas of the brain [189], it was effectively used for over a decade in motor rehabilitation treatments, and provides immediate stability in motor control rather than through a gradual learning process [190].

The temporal properties are scarcely investigated in spontaneous BCI, while they are essential for SSEP/ERP. I have not yet explored them in the literature on affordance, however they seem to be quite important for learning in general (in non-BCI contexts) [190, 191], and thus deserve to be seriously considered when designing user training protocols in all paradigms.

4.4.2. Object Function The perception (affordance) of object function (in visual, auditory or haptic modality) that can depend on socio-cultural experience (semantic knowledge) can be:

Manipulability, such as a ball [55], a row paddle [49] versus a penguin [56], worm [109] or a car [176] (although a wheel of a car is manipulable) etc; the sound of an object being manipulated versus an abstract, e.g. nature or synthetic sound;

Congruence, e.g. seeing or hearing the imagined object (action performed on it) the way one is imagining it $[57,174,179,180]$; or the object flashing is the one intended to be selected [60], and so on.

Familiarity, an object or sound can be familiar passively (e.g., music heard before, as in the SSVEP application [18]), or actively (e.g., practiced by the user, as walking in MI [180]) which would trigger mirror neurons (c.f. affordance literature, section 4).

Aesthetics, observing objects that are aesthetically pleasing increased acceptability of BCI [175], and are shown to relate with flow state, i.e., state of immersion, control and pleasure, which in turn correlates positively with BCI performance [56].

Synchronicity, background stimuli can be in or out of sync with the strategy performed, e.g., background music in [56] influenced motor imagery BCI performance. This feature could be also seen as a temporal congruence between the mental strategy and the background display.

Realness, multimodal cues and/or feedback 
which are realistic, proprioceptive (or embodied [47, 192]) can facilitate the transfer from training to real life applications [131, 193]. In VR literature, realness is also referred to as vividness, as real-life context naturally contains abundant, multimodal or perceptually rich stimuli [194].

These categories of task form are necessary for future task designs. It is the first step to acknowledging the influence of different stimuli on users as observers, which activate various neurophysiological pathways that in turn could modify system performance.

\section{Challenges}

After this extensive review there are still some challenges that remain to be considered as future work when designing BCI user training protocols. A few are mentioned in the following.

Introductory training. We could think of useful ways to improve the introductory training to best fit the user needs. For instance, how long should it last, where should it take place, could it be adaptive as well? It could be promising to adapt the instructions given before the interaction, for example to ask for user's preference over conceptual/ procedural/ interactive models; or to combine them depending on user understanding.

The power of sound. Even simple tones, high versus low pitch can influence size anticipation in grasping [195]. Auditory cues as ecological or everyday sounds as for instance footsteps proved useful for guiding human actions, influencing not only performance but significantly reducing variability in stride length for Parkinson's disease patients [160]. Additionally, response time is higher in auditory than in visual cues, compared in non BCI contexts [184, 196].

Authors in [190] mention a clinical motor rehabilitation model based on auditory-motor research from [197], within this model, auditory rhythmic signals as external stimuli can facilitate temporal muscular control of movement patterns by: (1) influencing timing and potentiation of motor neuron discharge, (2) decreasing muscular fatigue sensation, (3) facilitating automatized movement performance through the temporal predictability of its timing cues, (4) improving reaction time and response quality through facilitated response anticipation, and (5) providing auditory feedback for proprioceptive control mechanisms. [190]

In sum, there is great potential in sound, however it must be very carefully designed (e.g. congruent and in sync) as it can be as disruptive as it can be helpful.

Tangible affordance. Shape and material affordance on user experience are briefly mentioned
[138], while dynamic tangible affordance is a promising interface modality in HCI [139]. Without entering into detail, such literature could bring ideas on designing BCI haptic interfaces which are shown very promising (thoroughly reviewed in [198]).

Stimuli duration. I am not aware of an example where objects remain displayed rather than being replaced with novel feedback. For example, the bar size changes (the previous size being replaced with a new one), i.e. feedback is the mapping of the newly acquired EEG signal feature. But we could imagine a feedback where the history of classified data would remain in a multidimensional space. It could be useful to perceive the history of one's performance in time, but in that case, metrics such as classification accuracy would not suffice.

\section{Conclusion}

This paper contains an extensive review of existing training methods for most well-known BCI paradigms, SSEP, ERP and SMR, in order to support the need for protocol design standardization. Namely, the inconsistent instructions as well as task designs showed to modulate neural pathways and BCI performance in the literature.

With the help from other fields, such as HCI, psychology and education which has dwelt on designing more efficient user training a long time ago, three types of HCI user training are listed, and they are adjusted for BCI user introductory training. Those would use: (1) conceptual, i.e., explanations of underlying concepts of BCI, (2) procedural, i.e., "how-to" techniques to achieve high performances, or (3) interactive models, i.e., self-paced familiarization through trial-and-error, or free to explore a BCI without any evaluation. Preferably all three types are to be used during an introductory training, and in the proposed order. For instance, conceptual training would enable a correct initial formation of mental models (referred in BCI as mental strategies [109]), procedural would provide techniques to refine the strategies that best fit each individual, while interactive training would enable familiarization with the system. In HCI literature it is suggested not to start with Interaction, but rather with the conceptual model [107].

The task design is explained using a theoretical task model (extended from [95]), and filled with subcategories that are derived from perceptual affordance. Namely, those sub-categories belong to the task form and are: (1) qualitative, spatial or temporal properties; and (2) functions such as congruence, manipulability, aesthetics, realness, and synchronicity. It is important to note that choices made for a task design depend 
on the purpose of BCI application and on known neurophysiological reactions to stimuli, e.g. typically for the purpose of neuro-rehabilitation one would use SMR paradigm, motor imagery strategy in haptic, proprioceptive modality [198] and continuous form for the feedback [181], and if users were BCI naives, assistance would be proposed through positive feedback [53] for instance.

While some recommendations for user training are mentioned through literature review, it is not the sole purpose of this paper. Clear terms and categories are introduced here, that would enable an accountability of most effects external stimuli can have on neural activity. This paper serves mainly to inspire the BCI community to describe their protocols in proposed standardized terms, without omitting the reward system nor type of introduction used. When designing the task, I invite them to acknowledge the potential effects of rich, game-like environments on neural pathways and performance.

\section{Acknowledgments}

This paper takes part of a research done during my PhD thesis (2016-2019) which was mainly funded by BCI-Lift French national Inria project, and partially by Brain-Conquest project, grant ERC-2016-STG-714567.

\section{Bibliography}

[1] G. Dornhege, J. d. R. Millán, T. Hinterberger, D. McFarland, K.-R. Müller, et al. Toward brain-computer interfacing. Vol. 63. MIT press Cambridge, MA, 2007.

[2] C. Arrouët, M. Congedo, J.-E. Marvie, F. Lamarche, A. Lécuyer, and B. Arnaldi. "Open-ViBE: a three dimensional platform for real-time neuroscience". In: Journal of Neurotherapy 9.1 (2005), pp. 3-25.

[3] R. Leeb, D. Friedman, G. R. Müller-Putz, R. Scherer, M. Slater, and G. Pfurtscheller. "Self-paced (asynchronous) BCI control of a wheelchair in virtual environments: a case study with a tetraplegic". In: Computational intelligence and neuroscience 2007 (2007).

[4] L. A. Farwell and E. Donchin. "Talking off the top of your head: toward a mental prosthesis utilizing event-related brain potentials". In: Electroencephalography and clinical Neurophysiology 70.6 (1988), pp. 510-523.

[5] S. R. Soekadar, N. Birbaumer, M. W. Slutzky, and L. G. Cohen. "Brain-machine interfaces in neurorehabilitation of stroke". In: Neurobiology of disease 83 (2015), pp. 172179 .

[6] B. van de Laar, H. Gürkök, D. P.-O. Bos, M. Poel, and A. Nijholt. "Experiencing BCI control in a popular computer game". In: IEEE Transactions on Computational Intelligence and AI in Games 5.2 (2013), pp. 176-184.

[7] T. Gateau, H. Ayaz, and F. Dehais. "In silico vs. over the clouds: on-the-fly mental state estimation of aircraft pilots, using a functional near infrared spectroscopy based passive-BCI". In: Frontiers in human neuroscience 12 (2018), p. 187.
[8] J.-M. Batail, S. Bioulac, F. Cabestaing, C. Daudet, D. Drapier, M. Fouillen, et al. "EEG neurofeedback research: A fertile ground for psychiatry?" In: L'Encéphale 45.3 (2019), pp. 245-255.

[9] J. Wolpaw and E. W. Wolpaw. Brain-computer interfaces: principles and practice. OUP USA, 2012.

[10] B. Z. Allison and C. Neuper. "Could anyone use a BCI?" In: Brain-computer interfaces. Springer, 2010, pp. 35-54.

[11] F. Lotte, F. Larrue, and C. Mühl. "Flaws in current human training protocols for spontaneous brain-computer interfaces: lessons learned from instructional design". In: Frontiers in human neuroscience 7 (2013), p. 568.

[12] C. Sannelli, C. Vidaurre, K.-R. Müller, and B. Blankertz. "A large scale screening study with a SMR-based BCI: Categorization of BCI users and differences in their SMR activity". In: PLoS One 14.1 (2019), e0207351.

[13] R. Lorenz, J. Pascual, B. Blankertz, and C. Vidaurre. "Towards a holistic assessment of the user experience with hybrid BCIs". In: Journal of neural engineering 11.3 (2014), p. 035007.

[14] E Baykara, C. Ruf, C Fioravanti, I Käthner, N Simon, S. Kleih, et al. "Effects of training and motivation on auditory P300 brain-computer interface performance". In: Clinical Neurophysiology 127.1 (2016), pp. 379-387.

[15] T. Nierhaus, C. Vidaurre, C. Sannelli, K.-R. Mueller, and A. Villringer. "Immediate brain plasticity after one hour of brain-computer interface (BCI)". In: The Journal of physiology (2019).

[16] S. Halder, T. Leinfelder, S. M. Schulz, and A. Kübler. "Neural mechanisms of training an auditory event-related potential task in a brain-computer interface context". In: Human brain mapping 40.8 (2019), pp. 2399-2412.

[17] A. Evain, F. Argelaguet, N. Roussel, G. Casiez, and A. Lécuyer. "Can i think of something else when using a BCI? Cognitive demand of an SSVEP-based BCI". In: Proceedings of the 2017 CHI Conference on Human Factors in Computing Systems. 2017, pp. 5120-5125.

[18] L. Psotta, A. Rezeika, and I. Volosyak. "Investigating the Influence of Background Music on the Performance of an SSVEP-based BCI". In: 2019 IEEE International Conference on Systems, Man and Cybernetics (SMC). IEEE. 2019, pp. 4187-4193.

[19] E. Maby. "Technical Requirements for High-quality EEG Acquisition". In: Brain-Computer Interfaces 2: Technology and Applications (2016), pp. 143-161.

[20] S. Enriquez-Geppert, R. J. Huster, and C. S. Herrmann. "EEG-Neurofeedback as a Tool to Modulate Cognition and Behavior: A Review Tutorial". In: Frontiers in Human Neuroscience 11 (2017), p. 51.

[21] T. Ros, S. Enriquez-Geppert, V. Zotev, K. D. Young, G. Wood, S. Whitfield-Gabrieli, et al. Consensus on the reporting and experimental design of clinical and cognitive-behavioural neurofeedback studies (CRED-nf checklist). 2020.

[22] N. Leeuwis and M. Alimardani. "High Aptitude Motor Imagery BCI Users Have Better Visuospatial Memory". In: arXiv preprint arXiv:2010.02026 (2020).

[23] L Botrel, L Acqualagna, B Blankertz, and A Kübler. "Short progressive muscle relaxation or motor coordination training does not increase performance in a brain-computer interface based on sensorimotor rhythms (SMR)". In: International Journal of Psychophysiology 121 (2017), pp. 29-37. 
[24] L.-F. Tan, Z. Dienes, A. Jansari, and S.-Y. Goh. "Effect of mindfulness meditation on brain-computer interface performance". In: Consciousness and cognition 23 (2014), pp. $12-21$.

[25] A. Randolph, S. Karmakar, and M. Jackson. "Towards predicting control of a brain-computer interface". In: ICIS 2006 Proceedings (2006), p. 53.

[26] D. Zapała, E. Zabielska-Mendyk, P. Augustynowicz, A. Cudo, M. Jaśkiewicz, M. Szewczyk, et al. "The effects of handedness on sensorimotor rhythm desynchronization and motor-imagery BCI control". In: Scientific reports 10.1 (2020), pp. 1-11.

[27] E. M. Hammer, S. Halder, S. C. Kleih, and A. Kübler. "Psychological predictors of visual and auditory P300 brain-computer interface performance". In: Frontiers in neuroscience 12 (2018), p. 307.

[28] D. Zapała, M. Małkiewicz, P. Francuz, M. Kołodziej, and A. Majkowski. "Temperament Predictors of Motor Imagery Control in BCI". In: Journal of Psychophysiology (2019).

[29] T. Kaufmann, C. Vögele, S. Sütterlin, S. Lukito, and A. Kübler. "Effects of resting heart rate variability on performance in the P300 brain-computer interface". In: International journal of psychophysiology 83.3 (2012), pp. 336-341.

[30] M. Grosse-Wentrup and B. Schölkopf. "High gammapower predicts performance in sensorimotor-rhythm brain-computer interfaces". In: Journal of neural engineering 9.4 (2012), p. 046001.

[31] L. Botrel and A. Kübler. "Reliable predictors of SMR BCI performance-Do they exist?" In: 2018 6th International Conference on Brain-Computer Interface (BCI). IEEE. 2018, pp. 1-3.

[32] C. Benaroch, C. Jeunet, and F. Lotte. "Are users' traits informative enough to predict/explain their mentalimagery based BCI performances?" In: 2019.

[33] R. Vlek, J.-P. van Acken, E. Beursken, L. Roijendijk, and P. Haselager. "BCI and a User's Judgment of Agency". In: Brain-Computer-Interfaces in their ethical, social and cultural contexts. Springer, 2014, pp. 193-202.

[34] H.-J. Hwang, K. Kwon, and C.-H. Im. "Neurofeedbackbased motor imagery training for brain-computer interface (BCI)". In: Journal of neuroscience methods 179.1 (2009), pp. 150-156.

[35] A. Battison, M. Schlussel, T. Fuller, Y.-C. Yu, and L. Gabel. "Effectiveness of subject specific instruction on mu-based brain-computer interface training". In: 2015 41st Annual Northeast Biomedical Engineering Conference (NEBEC). IEEE. 2015, pp. 1-2.

[36] M. Daeglau, C. Zich, R. Emkes, J. Welzel, S. Debener, and C. Kranczioch. "Investigating Priming Effects of Physical Practice on Motor Imagery-Induced EventRelated Desynchronization". In: Frontiers in Psychology 11 (2020), p. 57.

[37] K. E. Sheets, D. Ryan, and E. W. Sellers. "The effect of task based motivation on BCI performance: a preliminary outlook". In: Proceedings of the 6th International BrainComputer Interface Conference, Graz University of Technology Publishing House. 2014.

[38] S. C. Kleih and A. Kübler. "Empathy, motivation, and P300 BCI performance". In: Frontiers in human neuroscience 7 (2013), p. 642.

[39] C. Neuper, R. Scherer, M. Reiner, and G. Pfurtscheller. "Imagery of motor actions: Differential effects of kinesthetic and visual-motor mode of imagery in singletrial EEG". In: Cognitive brain research 25.3 (2005), pp. $668-677$.
[40] B. Zoefel, R. J. Huster, and C. S. Herrmann. "Neurofeedback training of the upper alpha frequency band in EEG improves cognitive performance". In: Neuroimage 54.2 (2011), pp. 1427-1431.

[41] W. Nan, J. P. Rodrigues, J. Ma, X. Qu, F. Wan, P.-I. Mak, et al. "Individual alpha neurofeedback training effect on short term memory". In: International journal of psychophysiology 86.1 (2012), pp. 83-87.

[42] E. Hardman, J. Gruzelier, K. Cheesman, C. Jones, D. Liddiard, H. Schleichert, and N. Birbaumer. "Frontal interhemispheric asymmetry: self regulation and individual differences in humans". In: Neuroscience Letters 221.2-3 (1997), pp. 117-120.

[43] R. Scherer, J. Faller, E. V. Friedrich, E. Opisso, U. Costa, A. Kübler, and G. R. Müller-Putz. "Individually adapted imagery improves brain-computer interface performance in end-users with disability". In: PloS one 10.5 (2015), e0123727.

[44] D. P.-O. Bos, B. Reuderink, B. van de Laar, H. Gürkök, C. Mühl, M. Poel, et al. "Brain-computer interfacing and games". In: Brain-computer interfaces. Springer, 2010, pp. $149-178$.

[45] F. Putze, A. Vourvopoulos, A. Lécuyer, D. Krusienski, S. B. i Badia, T. Mullen, and C. Herff. "BrainComputer Interfaces and Augmented/Virtual Reality". In: Frontiers in Human Neuroscience 14 (2020).

[46] F. Lotte, J. Faller, C. Guger, Y. Renard, G. Pfurtscheller, A. Lécuyer, and R. Leeb. "Combining BCI with virtual reality: towards new applications and improved BCI". In: Towards Practical Brain-Computer Interfaces. Springer, 2012, pp. 197-220.

[47] N. Braun, R. Emkes, J. D. Thorne, and S. Debener. "Embodied neurofeedback with an anthropomorphic robotic hand". In: Scientific reports 6 (2016), p. 37696.

[48] F. Škola and F. Liarokapis. "Embodied VR environment facilitates motor imagery brain-computer interface training". In: Computers \& Graphics 75 (2018), pp. 5971.

[49] A. Vourvopoulos, A. Ferreira, and S. B. i Badia. "NeuRow: An Immersive VR Environment for MotorImagery Training with the Use of Brain-Computer Interfaces and Vibrotactile Feedback". In: International Conference on Physiological Computing Systems. Vol. 2. SCITEPRESS. 2016, pp. 43-53.

[50] A. Ramos-Murguialday, M. Schürholz, V. Caggiano, M. Wildgruber, A. Caria, E. M. Hammer, et al. "Proprioceptive feedback and brain computer interface (BCI) based neuroprostheses". In: PloS one 7.10 (2012), e47048.

[51] L. Bonnet, F. Lotte, and A. Lécuyer. "Two brains, one game: design and evaluation of a multiuser $\mathrm{BCI}$ video game based on motor imagery". In: IEEE Transactions on Computational Intelligence and $A I$ in games 5.2 (2013), pp. 185-198.

[52] M. Daeglau, F. Wallhoff, S. Debener, I. S. Condro, C. Kranczioch, and C. Zich. "Challenge Accepted? Individual Performance Gains for Motor Imagery Practice with Humanoid Robotic EEG Neurofeedback". In: Sensors 20.6 (2020), p. 1620.

[53] A. Kübler, B. Kotchoubey, J. Kaiser, J. R. Wolpaw, and N. Birbaumer. "Brain-computer communication: Unlocking the locked in." In: Psychological bulletin 127.3 (2001), p. 358.

[54] Á. Barbero and M. Grosse-Wentrup. "Biased feedback in brain-computer interfaces". In: Journal of neuroengineering and rehabilitation 7.1 (2010), p. 34. 
[55] M. Alimardani, S. Nishio, and H. Ishiguro. "Effect of biased feedback on motor imagery learning in BCI-teleoperation system." In: Frontiers in systems neuroscience 8.April (2014), p. 52.

[56] J. Mladenović, J. Frey, M. Bonnet-Save, J. Mattout, and F. Lotte. "The impact of flow in an EEG-based brain computer interface". In: Graz BCI conference (2017).

[57] J. Mladenović. "Computational Modeling of User States and Skills for Optimizing BCI Training Tasks". PhD thesis. 2019

[58] E. Maby, M. Perrin, O. Bertrand, G. Sanchez, and J. Mattout. "BCI could make old two-player games even more fun: a proof of concept with "Connect Four"". In: Advances in Human-Computer Interaction 2012 (2012).

[59] I. Cruz, C. Moreira, M. Poel, H. Ferreira, and A. Nijholt. "Kessel Run-A Cooperative Multiplayer SSVEP BCI Game". In: International Conference on Intelligent Technologies for Interactive Entertainment. Springer. 2017, pp. 77-95.

[60] A. Y. Kaplan, S. L. Shishkin, I. P. Ganin, I. A. Basyul, and A. Y. Zhigalov. "Adapting the P300-based braincomputer interface for gaming: a review". In: IEEE Transactions on Computational Intelligence and AI in Games 5.2 (2013), pp. 141-149.

[61] R Beveridge, S. Wilson, and D. Coyle. "3D graphics, virtual reality, and motion-onset visual evoked potentials in neurogaming". In: Progress in brain research. Vol. 228. Elsevier, 2016, pp. 329-353.

[62] J. Qu, F. Wang, Z. Xia, T. Yu, J. Xiao, Z. Yu, et al. "A Novel Three-Dimensional P300 Speller Based on Stereo Visual Stimuli". In: IEEE Transactions on Human-Machine Systems (2018).

[63] M. Donnerer and A. Steed. "Using a P300 braincomputer interface in an immersive virtual environment". In: Presence: Teleoperators and Virtual Environments 19.1 (2010), pp. 12-24.

[64] J. D. Bayliss, S. A. Inverso, and A. Tentler. "Changing the P300 brain computer interface". In: CyberPsychology E) Behavior 7.6 (2004), pp. 694-704.

[65] F. Putze, D. Wei $\beta$, L.-M. Vortmann, and T. Schultz. "Augmented Reality Interface for Smart Home Control using SSVEP-BCI and Eye Gaze". In: 2019 IEEE International Conference on Systems, Man and Cybernetics $(S M C)$. IEEE. 2019, pp. 2812-2817.

[66] J. Jin, E. W. Sellers, and X. Wang. "Targeting an efficient target-to-target interval for P300 speller brain-computer interfaces". In: Medical \& biological engineering \& computing 50.3 (2012), pp. 289-296.

[67] T. Kaufmann, S. Schulz, C. Grünzinger, and A. Kübler. "Flashing characters with famous faces improves ERP-based brain-computer interface performance". In: Journal of neural engineering 8.5 (2011), p. 056016.

[68] H. Ninomiya, T. Onitsuka, C.-h. Chen, E. Sato, and N. Tashiro. "P300 in response to the subject's own face". In: Psychiatry and clinical neurosciences 52.5 (1998), pp. 519-522.

[69] I Berlad and H Pratt. "P300 in response to the subject's own name". In: Electroencephalography and Clinical Neurophysiology/Evoked Potentials Section 96.5 (1995), pp. $472-474$.

[70] C. Cinel, R. Poli, and L. Citi. "Possible sources of perceptual errors in P300-based speller paradigm". In: Proceedings of the 2nd International BCI Workshop and Training Course (2004).
[71] T. Verhoeven, P. Buteneers, J. Wiersema, J. Dambre, and P. Kindermans. "Towards a symbiotic brain-computer interface: exploring the application-decoder interaction". In: Journal of neural engineering 12.6 (2015), p. 066027.

[72] M. Salvaris and F. Sepulveda. "Visual modifications on the P300 speller BCI paradigm". In: Journal of neural engineering 6.4 (2009), p. 046011.

[73] B. Mainsah, G Reeves, L. Collins, and C. Throckmorton. "Optimizing the stimulus presentation paradigm design for the P300-based brain-computer interface using performance prediction". In: Journal of neural engineering 14.4 (2017), p. 046025.

[74] A. Évain, F. Argelaguet, G. Casiez, N. Roussel, and A. Lécuyer. "Do the stimuli of an SSVEP-based BCI really have to be the same as the stimuli used for training it?" In: Brain-Computer Interfaces 3.2 (2016), pp. 103-111.

[75] Z. Wang, Y. Yu, M. Xu, Y. Liu, E. Yin, and Z. Zhou. "Towards a hybrid BCI gaming paradigm based on motor imagery and SSVEP". In: International Journal of Human-Computer Interaction 35.3 (2019), pp. 197205.

[76] B. J. Edelman, J. Meng, N. Gulachek, C. C. Cline, and B. He. "Exploring cognitive flexibility with a noninvasive BCI using simultaneous steady-state visual evoked potentials and sensorimotor rhythms". In: IEEE Transactions on Neural Systems and Rehabilitation Engineering 26.5 (2018), pp. 936-947.

[77] M. Wang, I. Daly, B. Z. Allison, J. Jin, Y. Zhang, L. Chen, and X. Wang. "A new hybrid BCI paradigm based on P300 and SSVEP". In: Journal of neuroscience methods 244 (2015), pp. 16-25.

[78] D. J. McFarland, W. A. Sarnacki, and J. R. Wolpaw. "Electroencephalographic (EEG) control of threedimensional movement". In: Journal of neural engineering 7.3 (2010), p. 036007.

[79] L. Ramsey, M. Tangermann, S. Haufe, and B. Blankertz. "Practicing fast-decision BCI using a" goalkeeper" paradigm". In: BMC Neuroscience 10.1 (2009), P69.

[80] M. Arvaneh, I. H. Robertson, and T. E. Ward. "A P300-Based Brain-Computer Interface for Improving Attention". In: Frontiers in human neuroscience 12 (2019), p. 524.

[81] L. George, L. Bonnet, and A. Lécuyer. "Freeze the BCI until the user is ready: a pilot study of a BCI inhibitor". In: 5th International Brain-Computer Interface Workshop. 2011.

[82] S. Aliakbaryhosseinabadi, D. Farina, and N. MrachaczKersting. "Real-time neurofeedback is effective in reducing diversion of attention from a motor task in healthy individuals and patients with amyotrophic lateral sclerosis". In: Journal of Neural Engineering (2020).

[83] E. V. Friedrich, C. Neuper, and R. Scherer. "Whatever works: a systematic user-centered training protocol to optimize brain-computer interfacing individually". In: PloS one 8.9 (2013), e76214.

[84] A Eliseyev and T Aksenova. "Personalized adaptive instruction design (PAID) for brain-computer interface using reinforcement learning and deep learning: simulated data study". In: Brain-Computer Interfaces 6.1-2 (2019), pp. 36-48.

[85] G. Pfurtscheller, B. Z. Allison, G. Bauernfeind, C. Brunner, T. Solis Escalante, R. Scherer, et al. "The hybrid BCI". In: Frontiers in neuroscience 4 (2010), p. 3. 
[86] D. Zapała, P. Francuz, E. Zapała, N. Kopiś, P. Wierzgała, P. Augustynowicz, et al. "The impact of different visual feedbacks in user training on motor imagery control in BCI". In: Applied psychophysiology and biofeedback 43.1 (2018), pp. 23-35.

[87] S. Kleih, F Nijboer, S Halder, and A Kübler. "Motivation modulates the P300 amplitude during brain-computer interface use". In: Clinical Neurophysiology 121.7 (2010), pp. 1023-1031.

[88] A. Roc, L. Pillette, B. N'Kaoua, and F. Lotte. "Would Motor-Imagery based BCI user training benefit from more women experimenters?" In: 8th International BCI Conference. Graz, Austria, Sept. 2019.

[89] S. Brandl, L. Frølich, J. Höhne, K.-R. Müller, and W. Samek. "Brain-computer interfacing under distraction: an evaluation study". In: Journal of neural engineering 13.5 (2016), p. 056012.

[90] Z. Emami and T. Chau. "The effects of visual distractors on cognitive load in a motor imagery brain-computer interface". In: Behavioural brain research 378 (2020), p. 112240 .

[91] D. Zhu, J. Bieger, G. Garcia Molina, and R. M. Aarts. "A survey of stimulation methods used in SSVEP-based BCIs". In: Computational intelligence and neuroscience 2010 (2010).

[92] H. M. d. A. Leite, S. N. d. Carvalho, T. B. d. S. Costa R. Attux, H. H. Hornung, and D. S. Arantes. "Analysis of user interaction with a brain-computer interface based on steady-state visually evoked potentials: case study of a game". In: Computational intelligence and neuroscience 2018 (2018).

[93] J. R. Wolpaw, N. Birbaumer, D. J. McFarland, G. Pfurtscheller, and T. M. Vaughan. "Brain-computer interfaces for communication and control". In: Clinical neurophysiology 113.6 (2002), pp. 767-791.

[94] S. C. Kleih and A. Kübler. "Psychological factors influencing brain-computer interface (BCI) performance". In 2015 IEEE International Conference on Systems, Man, and Cybernetics. IEEE. 2015, pp. 3192-3196.

[95] J. Mladenović, J. Mattout, and F. Lotte. "A generic framework for adaptive EEG-based BCI training and operation". In: CRC Press, 2018.

[96] J. J. Gibson. "Visually controlled locomotion and visual orientation in animals". In: British journal of psychology 49.3 (1958), pp. 182-194.

[97] K. J. W. Craik. The nature of explanation. Vol. 445. CUP Archive, 1952

[98] P. N. Johnson-Laird. Mental models: Towards a cognitive science of language, inference, and consciousness. 6 . Harvard University Press, 1983.

[99] D. A. Norman. "Some observations on mental models". In: Mental models. Psychology Press, 2014, pp. 15-22.

[100] K. Friston. "The free-energy principle: a unified brain theory?" In: Nature reviews neuroscience 11.2 (2010), p. 127.

[101] R. E. Mayer. "Models for understanding". In: Review of educational research 59.1 (1989), pp. 43-64.

[102] S. K. Card. The psychology of human-computer interaction. CRC Press, 2018.

[103] J. Carroll and R Mack. "Learning to use a word processor: By doing, by thinking, and by knowing". In: Human factors in computer systems. Ablex Publishing Corporation Norwood, New Jersey. 1984, pp. 13-51.
[104] M. K. Sein, R. P. Bostrom, and L. Olfman. "Conceptual models in training novice users". In: Human-Computer Interaction-INTERACT'87. Elsevier, 1987, pp. 861-867.

[105] C. L. Borgman. "The user's mental model of an information retrieval system: an experiment on a prototype online catalog". In: International Journal of man-machine studies 24.1 (1986), pp. 47-64.

[106] M. K. Sein and R. P. Bostrom. "Individual differences and conceptual models in training novice users". In: Human-computer interaction 4.3 (1989), pp. 197-229.

[107] R. Santhanam and M. K. Sein. "Improving end-user proficiency: Effects of conceptual training and nature of interaction". In: Information Systems Research 5.4 (1994), pp. 378-399.

[108] C. Jeunet, B. N'Kaoua, S. Subramanian, M. Hachet, and F. Lotte. "Predicting mental imagery-based BCI performance from personality, cognitive profile and neurophysiological patterns". In: PloS one 10.12 (2015), e0143962.

[109] M. Autenrieth, S. E. Kober, C. Neuper, and G. Wood. "How much do strategy reports tell about the outcomes of neurofeedback training? A study on the voluntary upregulation of the sensorimotor rhythm". In: Frontiers in Human Neuroscience 14 (2020), p. 218.

[110] G. Marko and U. Wimmer. "The brain is like a musclethe brain is like a control center: Conceptualizing the brain in expert and popularized scientific discourses". In: The talking (2018), p. 393.

[111] R. J. Vlek, D. Steines, D. Szibbo, A. Kübler, M.-J. Schneider, P. Haselager, and F. Nijboer. "Ethical issues in brain-computer interface research, development, and dissemination". In: Journal of Neurologic Physical Therapy 36.2 (2012), pp. 94-99.

[112] R. Scherer, A. Schwarz, G. R. Müller-Putz, V. PammerSchindler, and M. L. Garcia. "Game-based BCI training: Interactive design for individuals with cerebral palsy". In: 2015 IEEE International Conference on Systems, Man, and Cybernetics. IEEE. 2015, pp. 3175-3180.

[113] T. Ros, J. Théberge, P. A. Frewen, R. Kluetsch, M. Densmore, V. D. Calhoun, and R. A. Lanius. "Mind over chatter: plastic up-regulation of the fMRI salience network directly after EEG neurofeedback". In: Neuroimage 65 (2013), pp. 324-335.

[114] E. M. Hammer, S. Halder, B. Blankertz, C. Sannelli, T. Dickhaus, S. Kleih, et al. "Psychological predictors of SMR-BCI performance". In: Biological psychology 89.1 (2012), pp. $80-86$.

[115] R. M. Gibson, S. Chennu, A. M. Owen, and D. Cruse. "Complexity and familiarity enhance singletrial detectability of imagined movements with electroencephalography". In: Clinical Neurophysiology 125.8 (2014), pp. 1556-1567.

[116] R. Sitaram, T. Ros, L. Stoeckel, S. Haller, F. Scharnowski, J. Lewis-Peacock, et al. "Closed-loop brain training: the science of neurofeedback". In: Nature Reviews Neuroscience 18.2 (2017), p. 86.

[117] D. M. Wegner, D. J. Schneider, S. R. Carter, and T. L. White. "Paradoxical effects of thought suppression." In: Journal of personality and social psychology 53.1 (1987), p. 5.

[118] S. Ahn, M. Ahn, H. Cho, and S. C. Jun. "Achieving a hybrid brain-computer interface with tactile selective attention and motor imagery". In: Journal of neural engineering 11.6 (2014), p. 066004. 
[119] C. Neuper, G. Müller, A. Kübler, N. Birbaumer, and G. Pfurtscheller. "Clinical application of an EEG-based brain-computer interface: a case study in a patient with severe motor impairment". In: Clinical neurophysiology 114.3 (2003), pp. 399-409.

[120] S. Perdikis, L. Tonin, S. Saeedi, C. Schneider, and J. d. R. Millán. "The Cybathlon BCI race: Successful longitudinal mutual learning with two tetraplegic users". In: PLoS biology 16.5 (2018), e2003787.

[121] J. Frey, M. Daniel, J. Castet, M. Hachet, and F. Lotte. "Framework for electroencephalography-based evaluation of user experience". In: Proceedings of the 2016 CHI Conference on Human Factors in Computing Systems. 2016, pp. 2283-2294.

[122] C. Jeunet, S. Debener, F. Lotte, J. Mattout, R. Scherer, and C. Zich. "Mind the traps! design guidelines for rigorous BCI experiments". In: Brain-Computer Interfaces Handbook. CRC Press, 2018, pp. 639-660.

[123] R. Scherer, J. Faller, P. Sajda, and C. Vidaurre. "EEG-based Endogenous Online Co-Adaptive BrainComputer Interfaces: Strategy for Success?" In: 2018 10th Computer Science and Electronic Engineering (CEEC). IEEE. 2018, pp. 299-304.

[124] S. Perdikis and J. d. R. Millan. "Brain-Machine Interfaces: A Tale of Two Learners". In: IEEE Systems, Man, and Cybernetics Magazine 6.3 (2020), pp. 12-19.

[125] A Myrden and T Chau. "Towards psychologically adaptive brain-computer interfaces". In: Journal of neural engineering 13.6 (2016), p. 066022.

[126] S. Perdikis, R. Leeb, and J d R Millán. "Context-aware adaptive spelling in motor imagery BCI". In: Journal of neural engineering 13.3 (2016), p. 036018.

[127] J. S. Müller, C. Vidaurre, M. Schreuder, F. C. Meinecke, P. Von Bünau, and K.-R. Müller. "A mathematical model for the two-learners problem". In: Journal of neural engineering 14.3 (2017), p. 036005.

[128] R. Scherer, A. Schloegl, F. Lee, H. Bischof, J. Janša, and G. Pfurtscheller. "The self-paced Graz brain-computer interface: methods and applications". In: Computational intelligence and neuroscience 2007 (2007).

[129] C. Neuper, R. Scherer, M. Reiner, and G. Pfurtscheller. "Imagery of motor actions: Differential effects of kinesthetic and visual-motor mode of imagery in singletrial EEG". In: Cognitive brain research 25.3 (2005), pp. $668-677$.

[130] J. T. Hart. "Autocontrol of EEG alpha." In: Psychophysiology (1968).

[131] T. Ono, A. Kimura, and J. Ushiba. "Daily training with realistic visual feedback improves reproducibility of event-related desynchronisation following hand motor imagery". In: Clinical Neurophysiology 124.9 (2013), pp. $1779-1786$.

[132] J. Cantillo-Negrete, R. I. Carino-Escobar, P. CarrilloMora, J. A. Barraza-Madrigal, and O. Arias-Carrión. "Robotic orthosis compared to virtual hand for BrainComputer Interface feedback". In: Biocybernetics and Biomedical Engineering 39.2 (2019), pp. 263-272.

[133] L. M. Alonso-Valerdi, F. Sepulveda, and R. A. Ramírez-Mendoza. "Perception and cognition of cues Used in synchronous Brain-computer interfaces Modify electroencephalographic Patterns of control Tasks". In: Frontiers in human neuroscience 9 (2015), p. 636.

[134] L. Pillette, C. Jeunet, B. Mansencal, R. N'kambou, B. N'Kaoua, and F. Lotte. "A physical learning companion for Mental-Imagery BCI User Training". In: International Journal of Human-Computer Studies 136 (2020), p. 102380.
[135] J. Mladenovic, J. Frey, M. Joffily, E. Maby, F. Lotte, and J. Mattout. "Active inference as a unifying, generic and adaptive framework for a P300-based BCI". In: Journal of Neural Engineering 17.1 (2020), p. 016054.

[136] B. Rivet, A. Souloumiac, V. Attina, and G. Gibert. "xDAWN algorithm to enhance evoked potentials: application to brain-computer interface". In: IEEE Transactions on Biomedical Engineering 56.8 (2009), pp. 2035-2043.

[137] F. Galán, M. Nuttin, E. Lew, P. W. Ferrez, G. Vanacker, J. Philips, and J. d. R. Millán. "A brainactuated wheelchair: asynchronous and non-invasive brain-computer interfaces for continuous control of robots". In: Clinical neurophysiology 119.9 (2008), pp. 2159-2169.

[138] D. Petrelli, A. Soranzo, L. Ciolfi, and J. Reidy. "Exploring the aesthetics of tangible interaction: experiments on the perception of hybrid objects". In: Proceedings of the TEI'16: Tenth International Conference on Tangible, Embedded, and Embodied Interaction. 2016, pp. 100-108.

[139] S. Follmer, D. Leithinger, A. Olwal, A. Hogge, and H. Ishii. "inFORM: dynamic physical affordances and constraints through shape and object actuation." In: Uist. Vol. 13. 10.1145. 2013, pp. 2501988-2502032.

[140] J. M. Franchak, D. J. van der Zalm, and K. E. Adolph. "Learning by doing: Action performance facilitates affordance perception". In: Vision research 50.24 (2010), pp. $2758-2765$.

[141] E. Symes, R. Ellis, and M. Tucker. "Visual object affordances: Object orientation". In: Acta psychologica 124.2 (2007), pp. 238-255.

[142] L. Desanghere and J. J. Marotta. "The influence of object shape and center of mass on grasp and gaze". In: Frontiers in psychology 6 (2015), p. 1537.

[143] P. Garrigan. "The effect of contour closure on shape recognition". In: Perception 41.2 (2012), pp. 221-235.

[144] N. F. Regenberg, M. Häfner, and G. R. Semin. "The groove move: Action affordances produce fluency and positive affect." In: Experimental psychology 59.1 (2012), p. 30.

[145] D. Löffler, L. Arlt, T. Toriizuka, R. Tscharn, and J. Hurtienne. "Substituting color for haptic attributes in conceptual metaphors for tangible interaction design". In: Proceedings of the TEI'16: Tenth International Conference on Tangible, Embedded, and Embodied Interaction. 2016, pp. 118-125.

[146] L. L. Chao and A. Martin. "Representation of manipulable man-made objects in the dorsal stream". In: Neuroimage 12.4 (2000), pp. 478-484.

[147] A. M. Proverbio. "Tool perception suppresses 10-12 Hz $\mu$ rhythm of EEG over the somatosensory area". In: Biological psychology 91.1 (2012), pp. 1-7.

[148] J. P. Gallivan, A. McLean, and J. C. Culham. "Neuroimaging reveals enhanced activation in a reach-selective brain area for objects located within participants' typical hand workspaces". In: Neuropsychologia 49.13 (2011), pp. 3710-3721.

[149] P. J. Rowe, C. Haenschel, M. Kosilo, and K. Yarrow. "Objects rapidly prime the motor system when located near the dominant hand". In: Brain and cognition 113 (2017), pp. 102-108.

[150] P. Cardellicchio, C. Sinigaglia, and M. Costantini. "The space of affordances: a TMS study". In: Neuropsychologia 49.5 (2011), pp. 1369-1372. 
[151] Y. Wamain, F. Gabrielli, and Y. Coello. "EEG $\mu$ rhythm in virtual reality reveals that motor coding of visual objects in peripersonal space is task dependent". In: Cortex 74 (2016), pp. 20-30.

[152] S. Schuch, A. P. Bayliss, C. Klein, and S. P. Tipper. "Attention modulates motor system activation during action observation: evidence for inhibitory rebound". In: Experimental Brain Research 205.2 (2010), pp. 235-249.

[153] S. Lakatos, S. McAdams, and R. Caussé. "The representation of auditory source characteristics: Simple geometric form". In: Perception $\&$ psychophysics 59.8 (1997), pp. 1180-1190.

[154] C. Carello, K. L. Anderson, and A. J. Kunkler-Peck. "Perception of object length by sound". In: Psychological science 9.3 (1998), pp. 211-214.

[155] A. J. Kunkler-Peck and M. Turvey. "Hearing shape." In: Journal of Experimental psychology: human perception and performance 26.1 (2000), p. 279.

[156] M. M. Houben, A. Kohlrausch, and D. J. Hermes. "Perception of the size and speed of rolling balls by sound". In: Speech communication 43.4 (2004), pp. 331345.

[157] S. Bleeck and N. O'Meara. "The perception of size and shape of resonant objects". In: Journal of Hearing Science (2014), pp. 1-12.

[158] E. Thoret, M. Aramaki, R. Kronland-Martinet, J.-L. Velay, and S. Ystad. "From sound to shape: Auditory perception of drawing movements." In: Journal of Experimental Psychology: Human Perception and Performance 40.3 (2014), p. 983.

[159] W. W. Gaver. "What in the world do we hear?: An ecological approach to auditory event perception". In: Ecological psychology 5.1 (1993), pp. 1-29.

[160] C. J Steenson, M Rodger, and W Matthew. "Bringing sounds into use: thinking of sounds as materials and a sketch of auditory affordances". In: The Open Psychology Journal 8.1 (2015).

[161] J.-P. VERNAT and M. S. Gordon. "Indirect interception actions by blind and sighted perceivers: The role of modality and tau". In: Scandinavian journal of psychology 52.1 (2011), pp. 83-92.

[162] A. Sedda, S. Monaco, G. Bottini, and M. A. Goodale. "Integration of visual and auditory information for hand actions: preliminary evidence for the contribution of natural sounds to grasping". In: Experimental brain research 209.3 (2011), pp. 365-374.

[163] U. Castiello, B. L. Giordano, C. Begliomini, C. Ansuini, and M. Grassi. "When ears drive hands: the influence of contact sound on reaching to grasp". In: PloS one 5.8 (2010).

[164] L Pizzamiglio, T Aprile, G Spitoni, S Pitzalis, E Bates, $\mathrm{S}$ D'amico, and F Di Russo. "Separate neural systems for processing action-or non-action-related sounds". In: Neuroimage 24.3 (2005), pp. 852-861.

[165] L. Aziz-Zadeh, M. Iacoboni, E. Zaidel, S. Wilson, and J. Mazziotta. "Left hemisphere motor facilitation in response to manual action sounds". In: European Journal of Neuroscience 19.9 (2004), pp. 2609-2612.

[166] A. Lahav, E. Saltzman, and G. Schlaug. "Action representation of sound: audiomotor recognition network while listening to newly acquired actions". In: Journal of Neuroscience 27.2 (2007), pp. 308-314.

[167] G. Buccino, S. Vogt, A. Ritzl, G. R. Fink, K. Zilles, H.-J. Freund, and G. Rizzolatti. "Neural circuits underlying imitation learning of hand actions: an event-related fMRI study". In: Neuron 42.2 (2004), pp. 323-334.
[168] O. Hauk, Y. Shtyrov, and F. Pulvermüller. "The sound of actions as reflected by mismatch negativity: rapid activation of cortical sensory-motor networks by sounds associated with finger and tongue movements". In: European Journal of Neuroscience 23.3 (2006), pp. 811821.

[169] J. Krueger. "Affordances and the musically extended mind". In: Frontiers in psychology 4 (2014), p. 1003.

[170] F. Cummins. "Rhythm as an affordance for the entrainment of movement". In: Phonetica 66.1-2 (2009), pp. $15-28$.

[171] F. Cuadrado, I. Lopez-Cobo, T. Mateos-Blanco, and A. Tajadura-Jiménez. "Arousing the sound: A field study on the emotional impact on children of arousing sound design and 3D audio spatialization in an audio story". In: Frontiers in Psychology 11 (2020), p. 737.

[172] G. Pfurtscheller and C. Neuper. "Motor imagery and direct brain-computer communication". In: Proceedings of the IEEE 89.7 (2001), pp. 1123-1134.

[173] C. Neuper, R. Scherer, S. Wriessnegger, and G. Pfurtscheller. "Motor imagery and action observation: modulation of sensorimotor brain rhythms during mental control of a brain-computer interface". In: Clinical neurophysiology 120.2 (2009), pp. 239-247.

[174] M. E. Thurlings, J. B. van Erp, A.-M. Brouwer, B. Blankertz, and P. Werkhoven. "Control-display mapping in brain-computer interfaces". In: Ergonomics 55.5 (2012), pp. 564-580.

[175] F. Nijboer. "Technology transfer of brain-computer interfaces as assistive technology: barriers and opportunities". In: Annals of physical and rehabilitation medicine 58.1 (2015), pp. 35-38.

[176] R. Ron-angevin. "Brain-computer interface: Changes in performance using virtual reality techniques". In: 449 (2009), pp. 123-127.

[177] F. Nijboer, I. Gunst, S. Von Hartlieb, D. McFarland, N. Birbaumer, and A Kübler. "A comparison between auditory and visual feedback of sensorimotor rhythms (SMR) for a Brain-computer interface (BCI) in healthy participants". In: Psychophysiology 43 (2006), S71-S71.

[178] I. Daly, D. Williams, F. Hwang, A. Kirke, A. Malik, E. Roesch, et al. "Investigating music tempo as a feedback mechanism for closed-loop BCI control". In: BrainComputer Interfaces 1.3-4 (2014), pp. 158-169.

[179] E. Tidoni, P. Gergondet, A. Kheddar, and S. M. Aglioti. "Audio-visual feedback improves the BCI performance in the navigational control of a humanoid robot". In: Frontiers in neurorobotics 8 (2014), p. 20.

[180] E. Christophe, J. Frey, R. Kronland-Martinet, J.-A. Micoulaud-Franchi, J. Mladenović, G. Mougin, et al. "Evaluation of a congruent auditory feedback for Motor Imagery BCI". In: International BCI meeting. 2018.

[181] C. Neuper, A. Schlögl, and G. Pfurtscheller. "Enhancement of left-right sensorimotor EEG differences during feedback-regulated motor imagery". In: Journal of Clinical Neurophysiology 16.4 (1999), pp. 373-382.

[182] T. Hinterberger, N. Neumann, M. Pham, A. Kübler, A. Grether, N. Hofmayer, et al. "A multimodal brain-based feedback and communication system". In: Experimental brain research 154.4 (2004), pp. 521-526.

[183] W. H. Teichner. "Recent studies of simple reaction time." In: Psychological bulletin 51.2 (1954), p. 128. 
[184] A. Jain, R. Bansal, A. Kumar, and K. Singh. "A comparative study of visual and auditory reaction times on the basis of gender and physical activity levels of medical first year students". In: International Journal of Applied and Basic Medical Research 5.2 (2015), p. 124.

[185] C. Guger, S. Daban, E. Sellers, C. Holzner, G. Krausz, R. Carabalona, et al. "How many people are able to control a P300-based brain-computer interface (BCI)?" In: Neuroscience letters 462.1 (2009), pp. 94-98.

[186] Z. Wu, Y. Lai, Y. Xia, D. Wu, and D. Yao. "Stimulator selection in SSVEP-based BCI". In: Medical engineering 83 physics 30.8 (2008), pp. 1079-1088.

[187] R. Scherer, F. Lee, A. Schlogl, R. Leeb, H. Bischof, and G. Pfurtscheller. "Toward self-paced brain-computer communication: navigation through virtual worlds". In IEEE Transactions on Biomedical Engineering 55.2 (2008), pp. 675-682.

[188] M. Thaut, G. Kenyon, M. Schauer, and G. McIntosh. "The connection between rhythmicity and brain function". In: IEEE Engineering in Medicine and Biology Magazine 18.2 (1999), pp. 101-108.

[189] S. L. Bengtsson, F. Ullen, H. H. Ehrsson, T. Hashimoto, T. Kito, E. Naito, et al. "Listening to rhythms activates motor and premotor cortices". In: cortex 45.1 (2009), pp. $62-71$.

[190] A. B. LaGasse and M. W. Hardy. "Rhythm, movement, and autism: using rhythmic rehabilitation research as a model for autism". In: Frontiers in integrative neuroscience 7 (2013), p. 19.

[191] R. Sigrist, G. Rauter, R. Riener, and P. Wolf. "Augmented visual, auditory, haptic, and multimodal feedback in motor learning: a review". In: Psychonomic bulletin 8 review 20.1 (2013), pp. 21-53.

[192] M. Alimardani, S. Nishio, and H. Ishiguro. "The importance of visual feedback design in BCIs; from embodiment to motor imagery learning". In: PloS one 11.9 (2016), e0161945.

[193] G. Edlinger and C. Guger. "Social environments, mixed communication and goal-oriented control application using a brain-computer interface". In: International conference on universal access in human-computer interaction. Springer. 2011, pp. 545-554.

[194] J. Steuer. "Defining virtual reality: Dimensions determining telepresence". In: Journal of communication 42.4 (1992), pp. 73-93.

[195] L. Rinaldi, C. Lega, Z. Cattaneo, L. Girelli, and N. F. Bernardi. "Grasping the sound: Auditory pitch influences size processing in motor planning." In: Journal of Experimental Psychology: Human Perception and Performance 42.1 (2016), p. 11.

[196] T. P. Ghuntla, H. B. Mehta, P. A. Gokhale, C. J. Shah, et al. "A comparison and importance of auditory and visual reaction time in basketball players". In: Saudi Journal of Sports Medicine 14.1 (2014), p. 35.

[197] M. H. Thaut. "Rhythmic intervention techniques in music therapy with gross motor dysfunctions." In: The Arts in Psychotherapy (1988).

[198] M. Fleury, G. Lioi, C. Barillot, and A. Lécuyer. "A Survey on the Use of Haptic Feedback for BrainComputer Interfaces and Neurofeedback". In: (2020). 